\title{
CONCURRENT TERNARY GALOIS-BASED COMPUTATION USING NANO-APEX MULTIPLEXING Nibs OF REgular THREE-DimensionaL NeTWORKS, PART I: BASICS
}

\author{
Anas N. Al-Rabadi \\ Department of Computer Engineering, The University of Jordan, Amman - Jordan \& \\ Department of Renewable Energy Engineering, Isra University - Jordan
}

\begin{abstract}
New implementations within concurrent processing using three-dimensional lattice networks via nano carbon-based field emission controlled-switching is introduced in this article. The introduced nano-based three-dimensional networks utilize recent findings in nano-apex field emission to implement the concurrent functionality of lattice networks. The concurrent implementation of ternary Galois functions using nano threedimensional lattice networks is performed by using carbon field-emission switching devices via nano-apex carbon fibers and nanotubes. The presented work in this part of the article presents important basic background and fundamentals with regards to lattice computing and carbon field-emission that will be utilized within the follow-up works in the second and third parts of the article. The introduced nano-based three-dimensional lattice implementations form new and important directions within three-dimensional design in nanotechnologies that require optimal specifications of high regularity, predictable timing, high testability, fault localization, self-repair, minimum size, and minimum power consumption.
\end{abstract}

\section{KEYWORDS}

Carbon nano-apex, Concurrent processing, Field emission, Lattice network, Regularity, Symmetric function.

\section{INTRODUCTION}

With future logic realization in technologies that are scaled down rapidly in size, the emphasis will be increasingly focused on the mutually linked issues of regularity, predictable timing, high testability, fast fault localization and self-repair [2], [3], [7], [31].

For the current leading technologies with the active-device count reaching the hundreds of millions, and more than $80 \%$ of circuit areas are occupied by local and global interconnects, the delay of interconnects is responsible for up to $50 \%$ or more of the total delay associated with a circuit [2], [31]. Interconnects will take even higher percent of area and delay within future technologies, which creates rising and increasing interests in cellular and regular circuits especially for deep submicron and nanotechnologies. For example, Fig. 1 illustrates trends for electrical signal delays for global interconnects with repeaters and without repeaters versus the local interconnects [2], [31]. 


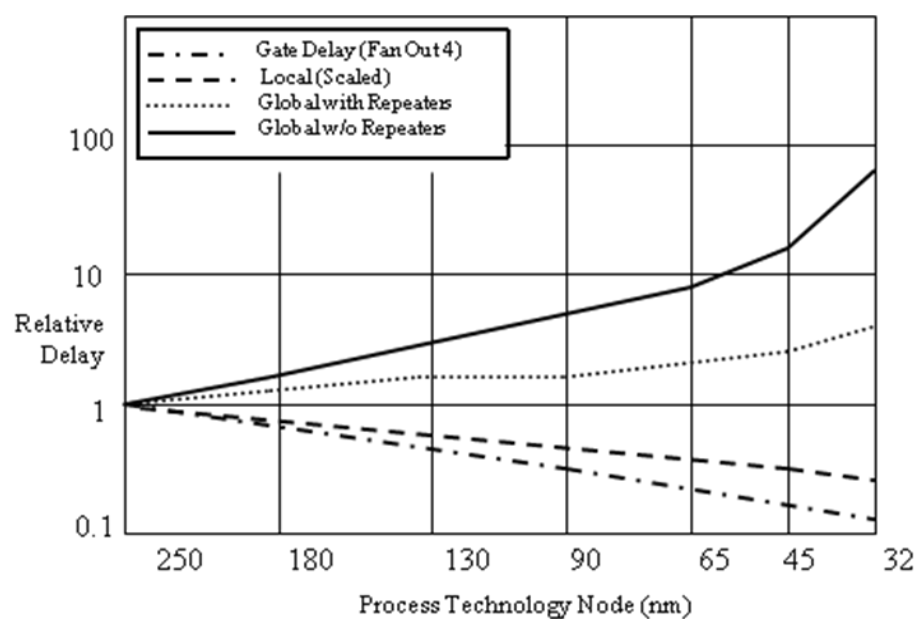

Figure 1. Delays for local and global interconnects versus the utilized feature size.

Lattice circuits [2], [3] generalize the ideas from the well-known regular circuits such as Fat Trees, Generalized PLAs, Maitra Cascades and Akers Arrays [1], into a more systematic framework which is closely related to the symmetry of functions and symmetric networks [2], [3], [28]. Realization of logic circuits in three-dimensional space can be very important for future technologies, as it shows that the best way is to synthesize combinational logic functions in a three-dimensional space where all local interconnections are of the same length and global interconnections are only inputs on parallel oblique planes (cf. Section 3). Moreover, three-dimensional cubical lattice circuits have special importance since three-dimensional crystal lattices exist where inter-related atoms that exist in a potential field are spaced on the corners of three-dimensional cubes, and thus the real potential of the physical implementation of the logically synthesized three-dimensional lattice networks using three-dimensional crystal lattices.

Nanotechnology is a new interdisciplinary field of research that cuts across several fields of engineering, chemistry, physics and biology, that analyzes and synthesizes systems in the nano scale (equal or less than $10^{-9} \mathrm{~m}$ ) such as nanoparticles, nanowires, nanosheets and carbon nanotubes (CNTs) [4], [5]-[7], [10], [11], [15]-[19], [22], [26], [29], [34], [36]-[38]. For example, nanocapsules that contain charged particles for better medical delivery has been proposed [34], methods for logic design that can be useful in nano integrated circuits have been presented [35], utilizing CNT field emission for scanning electron microscopy has been shown [11], the investigation of nanoelectronics has been presented [29], the potential uses of nanotubes in important future applications have been demonstrated [10], and the introduction of the important upcoming era of nanotechnology applications has been deeply manifested [19]. The CNT technology is one of several cutting-edge emerging technologies within nanotechnology that is showing high efficiency and very wide range of applications in several various fields in science and technology, where recent examples of such applications include TVs based on field-emission of CNTs that consume much less power, thinner and are of much higher resolution, and nanocircuits based on CNTs such as CNT Field Effect Transistors (FETs) that show high potential for consuming less power and to be much faster than the available silicon-based FETs.

Recently, carbon nanotubes have attracted much attention not only for their unique morphologies and relatively small dimensions, but also for their potential implementations in many current and 
emerging technologies [10], [16]-[18]. The CNT is made of graphite, where it has been observed that graphite can be formed within nano-scale in three different forms: (1) Carbon nanoball (or buckyball) which is a molecule that consists of 60 carbon atoms $\left(\mathrm{C}_{60}\right)$ that are arranged in the form of a soccer ball, (2) Carbon nanotube (CNT) which is a narrow strip of tiny sheet of graphite that comes mainly in two types of multi-wall CNT (MWCNT) where each CNT contains several hollow cylinders of carbon atoms nested inside each other and single-wall CNT (SWCNT) that is made of just a single layer of carbon atoms, and (3) Carbon nanocoil. Electrical implementations of basic logic gates such as the logic inverter using CNTs have been demonstrated [18], engineering CNT circuits using electrical breakdown has been shown [17], the utilization of CNT films for cathodebased implementations has been demonstrated [30], designing CNT multiplexer-based circuits and actuators has been shown [4]-[6], and the potential importance of CNTs for designing electronic circuits and systems has been proposed [16].

This first part of the article introduces basic background in concurrent processing using threedimensional lattice networks and basic carbon-based field emission characteristics that will be used in the second and third parts of the article within concurrent processing via field emission-based three-dimensional lattice networks. Figure 2 illustrates the hierarchical layout of the introduced system design methodology where layer 1 shows the underlying utilized fundamental Galois field algebra, layer 2 illustrates the field-emission physics which is used in the operation of the utilized devices, layer 3 shows the carbon-based technology which is used in the synthesis of the field emission - based switching devices, and layers 4 - 5 show the corresponding hierarchical implementation into the system level.

\begin{tabular}{|c|}
\hline Three-Dimensional Lattice Realizations \\
\hline Field Emission-Based Circuits \\
\hline Carbon-Based Field Emission Devices \\
\hline Field-Emission Physics \\
\hline Galois Algebra \\
\hline
\end{tabular}

Figure 2. The introduced and utilized system implementation hierarchy.

As previously stated, the presented work in this part will be utilized within the follow-up works in the second and third parts of the article for the design of concurrent nano-based three-dimensional networks. The research findings and implementation in this article are new and original and are performed for the concurrent implementation of ternary functions using nano three-dimensional networks using carbon field-emission switching devices via nano-apex carbon fibers and nanotubes.

The remainder of this article is organized as follows: Basic backgrounds on two-dimensional and three-dimensional lattice networks are given in Sections 2 and 3, respectively. Basics of carbonbased nanotubes and nanotips and their corresponding field emission characteristics are presented in Section 4. Conclusions are presented in Section 5.

\section{TWO-DIMENSIONAL LATTICE NETWORKS}

The binary hierarchy of families of canonical forms [2], [23], [32] and the corresponding decision diagrams [13] are based on three basic functional expansions: Shannon, positive Davio and negative Davio expansions, which are given below respectively:

$$
f\left(x_{1}, x_{2}, \ldots, x_{n}\right)=x_{1}^{\prime} \cdot f_{0}\left(x_{1}, x_{2}, \ldots, x_{n}\right) \oplus x_{1} \cdot f_{1}\left(x_{1}, x_{2}, \ldots, x_{n}\right)
$$




$$
\begin{gathered}
f\left(x_{1}, x_{2}, \ldots, x_{n}\right)=1 \cdot f_{0}\left(x_{1}, x_{2}, \ldots, x_{n}\right) \oplus x_{1} \cdot f_{2}\left(x_{1}, x_{2}, \ldots, x_{n}\right) \\
f\left(x_{1}, x_{2}, \ldots, x_{n}\right)=1 \cdot f_{1}\left(x_{1}, x_{2}, \ldots, x_{n}\right) \oplus x_{1}^{\prime} \cdot f_{2}\left(x_{1}, x_{2}, \ldots, x_{n}\right)
\end{gathered}
$$

Where $f_{0}\left(x_{1}, x_{2}, \ldots, x_{n}\right)=f\left(0, x_{2}, \ldots, x_{n}\right)=f_{0}$ is the negative cofactor of variable $x_{1}, f_{1}\left(x_{1}, x_{2}, \ldots, x_{n}\right)=$ $f\left(1, x_{2}, \ldots, x_{n}\right)=f_{1}$ is the positive cofactor of variable $x_{1}$, and $f_{2}\left(x_{1}, x_{2}, \ldots, x_{n}\right)=f\left(0, x_{2}, \ldots, x_{n}\right) \oplus f(1$, $\left.x_{2}, \ldots, x_{n}\right)=f_{0} \oplus f_{1}$. All operations in Equations (1) - (3) are performed using Boolean algebra, i.e., $\oplus$ is Boolean XOR, and · is Boolean multiplication.

Multiple-valued spectral methods are used in many applications in synthesis, analysis, testing, classification and verification of logic circuits and systems [27]. Because Galois field proved to possess desired properties in many applications such as test, communications and signal processing, the developments of three-dimensional lattice networks will be conducted on the corresponding Galois field (GF) algebraic structures. Radix three Galois field (GF(3)) addition and multiplication are defined in Tables 1(a) and 1(b), respectively.

Table 1. Third radix Galois field (a) GF(3) addition, and (b) GF(3) multiplication.

(a)

\begin{tabular}{|l|l|l|l|}
\hline+ & $\mathbf{0}$ & $\mathbf{1}$ & $\mathbf{2}$ \\
\hline $\mathbf{0}$ & 0 & 1 & 2 \\
\hline $\mathbf{1}$ & 1 & 2 & 0 \\
\hline $\mathbf{2}$ & 2 & 0 & 1 \\
\hline
\end{tabular}

(b)

\begin{tabular}{|l|l|l|l|}
\hline$*$ & $\mathbf{0}$ & $\mathbf{1}$ & $\mathbf{2}$ \\
\hline $\mathbf{0}$ & 0 & 0 & 0 \\
\hline $\mathbf{1}$ & 0 & 1 & 2 \\
\hline $\mathbf{2}$ & 0 & 2 & 1 \\
\hline
\end{tabular}

A literal is a function of a single variable. 1-Reduced Post literal (1-RPL) is defined as:

$$
{ }^{i} x=1 \text { iff } x=i \text { else }{ }^{i} x=0
$$

For example ${ }^{0} x,{ }^{1} x$, and ${ }^{2} x$ are the zero, first, and second polarities of the 1-RPL, respectively [2]. Also, the ternary shifts of variable $x$ are defined as: $x$ with no shift, $x^{\prime}$ with one shift and $x^{\prime \prime}$ with two shifts (i.e., $x=x+0, x^{\prime}=x+1$, and $x^{\prime \prime}=x+2$, respectively), and $x$ can take any value in the set $\{0$, 1, 2\}. Ternary 1-RPL will be used in Section 3 to construct three-dimensional lattice networks by controlling the propagation of sub-functions in three-dimensions.

The concept of lattice networks for switching functions [2] involves three components: (1) expansion of a function that corresponds to the root (initial node) in the lattice which creates several successor nodes of the expanded node, (2) joining of several nodes of a decision tree level to a single node which is the reverse operation of the expansion process, and (3) regular geometry to which the nodes are mapped that guides which nodes of the level are to be joined.

While the realization of Boolean non-symmetric functions in Akers arrays requires an exponential growth of the repetition of variables in the worst case [1]-[2], the realization of Boolean nonsymmetric functions in lattice networks requires a linear growth of repetition of variables, and consequently one does not need to repeat the variables of non-symmetric functions too many times to realize such functions in lattice networks for most practical benchmarks [2].

Figure 3 illustrates, as an example, the geometry of a four-neighbor lattice network and joining operations on the nodes where each cell has two inputs and two outputs (i.e., four neighbors). The construction of the lattice network in Fig. 3 implements the following one possible convention of 
top-to-bottom expansion and left-to-right joining (i.e., left-to-right propagation of the corresponding correction functions in Figs. 3(c) and 3(d), respectively).

Definition 1. The function that is generated by joining two nodes (sub-functions) in a lattice network is called the joined function. The function that is generated in nodes other than the joining nodes, to preserve the functionality within the lattice network, is called the correction function.

Note that the lattices shown in Fig. 3 preserve the functionality of the corresponding sub-functions $f$ and $g$. This can be observed, for instance, in Fig. 3(b) as the negated variable $\left\{a^{\prime}\right\}$ will cancel the un-complemented variable $\{a\}$, when propagating the cofactors from the lower levels to the upper levels or vice versa, without the need for any correction functions to preserve the output functionality of the corresponding lattice network. This simple observation cannot be seen directly in Figs. 3(c) and 3(d), as the correction functions are involved to cancel the effect of the new joining nodes for the preservation of the functionality of the new lattice networks (these correction functions are shown in the extreme right leaves of the second level in Figs. 3(c) and 3(d), respectively).

It is shown [2], [28] that every function that is not symmetric can be symmetrized by repeating variables, and that a totally symmetric function can be obtained from an arbitrary non-symmetric function by the repetition of variables. Consequently, lattice networks and the symmetry of functions are very much related to each other. Example 1 will illustrate such very close relationship.

Example 1. For the following non-symmetric three-variable Boolean function $F=a \cdot b+a^{\prime} \cdot c$, by utilizing the joining rule that was presented in Fig. 3(b) for two-dimensional lattice network with binary Shannon nodes, one obtains the lattice network shown in Fig. 4.

One can note that without the repetition of variable(s) (e.g., variable $b$ in Fig. 4) $F$ cannot be produced by any lattice network. It is also to be noted that all internal nodes in Fig. 4 are two-to-one multiplexers (i.e., selectors). In Fig. 4, if one multiplies each leaf value, from left to right, with all possible bottom-up paths (from the leaves to the root $F$ ) and add them over Boolean algebra then one obtains the function $F$ (i.e., the root) as follows:

$$
\begin{aligned}
F & =\left(0 \cdot c^{\prime} \cdot \mathrm{b}^{\prime} \cdot \mathrm{a}^{\prime}\right)+\left(1 \cdot \mathrm{c}^{\prime} \cdot \mathrm{b}^{\prime} \cdot \mathrm{a}^{\prime}\right)+\left(0 \cdot \mathrm{c}^{\prime} \cdot \mathrm{b}^{\prime} \cdot \mathrm{a}^{\prime}\right)+\left(0 \cdot \mathrm{b}^{\prime} \cdot \mathrm{c} \cdot \mathrm{b}^{\prime} \cdot \mathrm{a}^{\prime}\right)+\left(1 \cdot \mathrm{b} \cdot \mathrm{c} \cdot \mathrm{b}^{\prime} \cdot \mathrm{a}^{\prime}\right)+\left(1 \cdot \mathrm{c}^{\prime} \cdot \mathrm{b} \cdot \mathrm{a}\right)+\left(1 \cdot \mathrm{c} \cdot \mathrm{b}^{\prime} \cdot \mathrm{a}\right) \\
& =\left(1 \cdot \mathrm{c}^{\prime} \cdot \mathrm{b}^{\prime} \cdot \mathrm{a}^{\prime}\right)+\left(1 \cdot \mathrm{b}^{\prime} \cdot \mathrm{c}^{\prime} \cdot \mathrm{b}^{\prime} \cdot \mathrm{a}^{\prime}\right)+\left(1 \cdot \mathrm{c}^{\prime} \cdot \mathrm{b} \cdot \mathrm{a}\right)+(1 \cdot \mathrm{c} \cdot \mathrm{b} \cdot \mathrm{a}) \\
& =a^{\prime} \cdot c+a \cdot b
\end{aligned}
$$

One can observe that in order to represent the non-symmetric function in Example 1 in the twodimensional lattice network, variable $b$ is repeated, where the nodes in Fig. 4 are Shannon nodes, which are merely two-input one-output multiplexers, whose output goes in two directions, with the set of variables $\{a, b, c\}$ operate as control signals. 


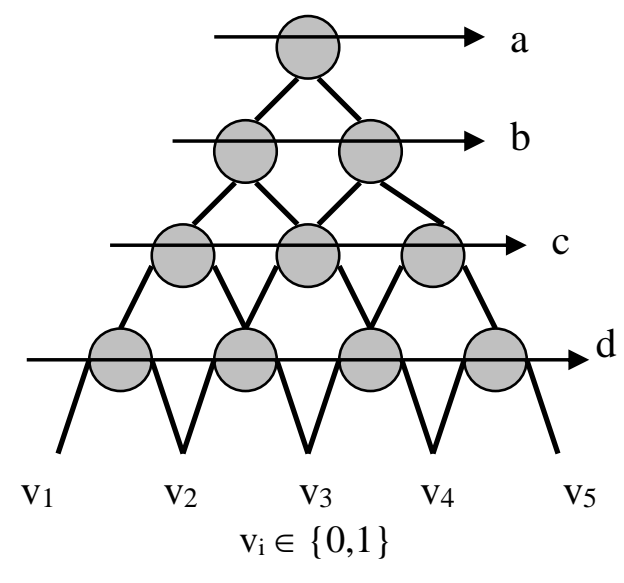

(a)

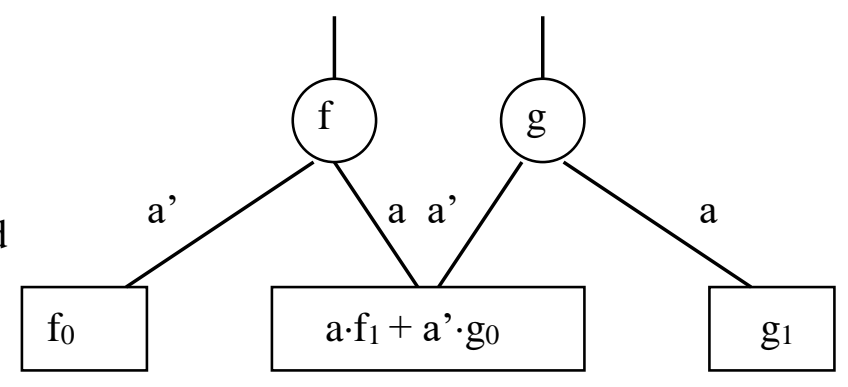

(b)

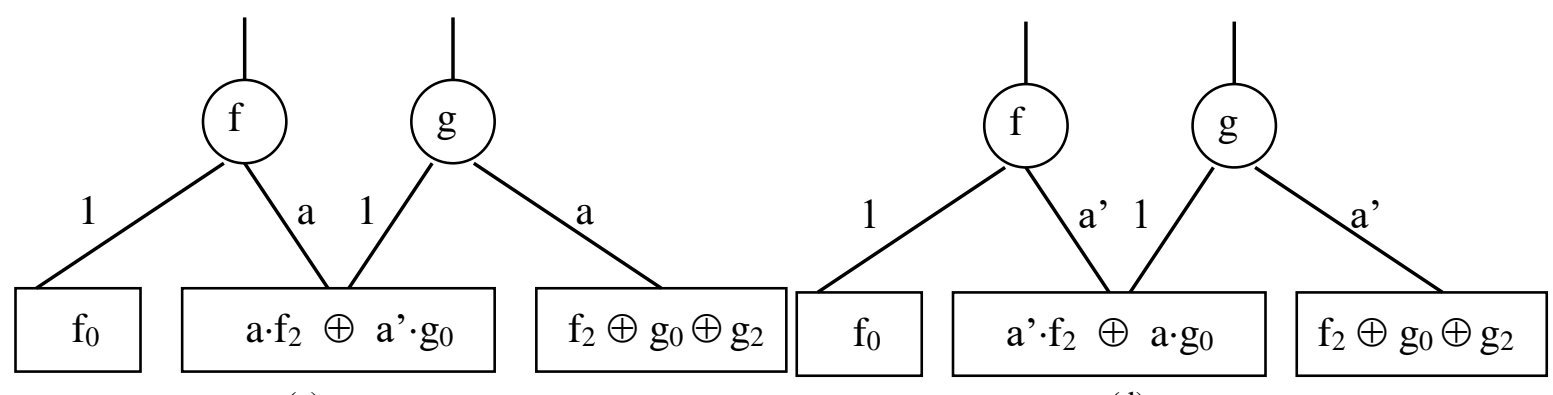

(c)

(d)

Figure 3. Two-dimensional binary lattice networks: (a) A two-dimensional 4-neighbor lattice network, (b) Shannon lattice network, (c) positive Davio lattice network, and (d) negative Davio lattice network.

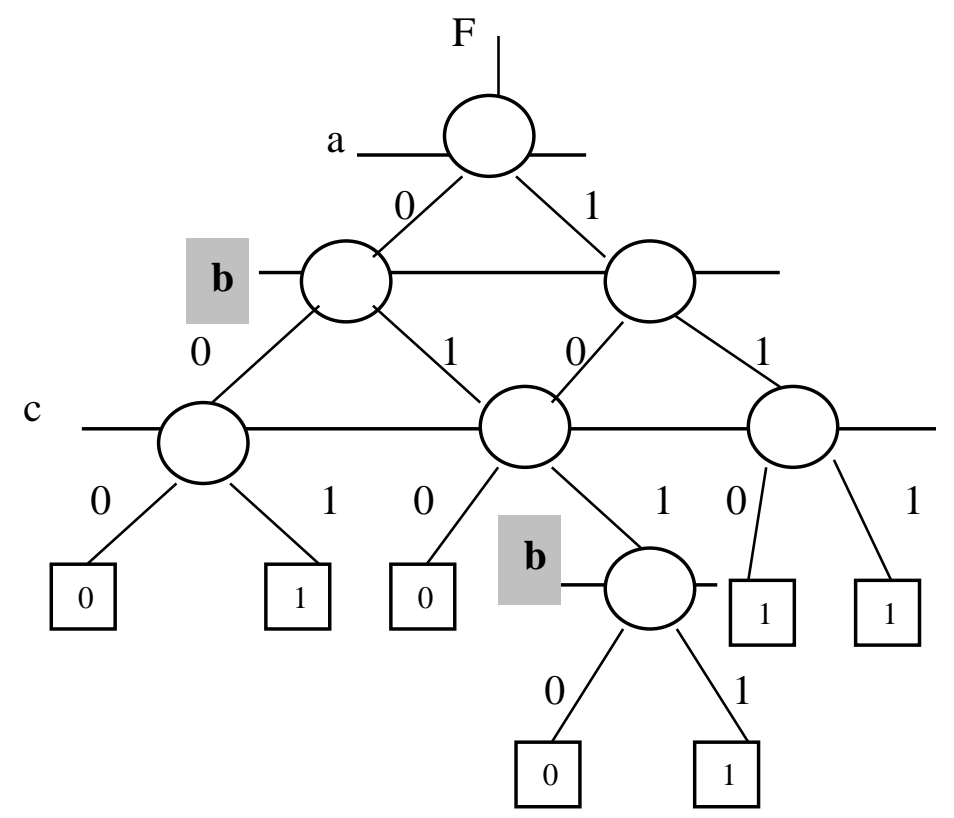

Figure 4. Two-dimensional Shannon lattice network for the corresponding non-symmetric function $F=a \cdot b+a^{\prime} \cdot c$. 
The results from this section will be generalized to the ternary logic in Section 3, and thus from two-dimensional space to three-dimensional space. It is important to prove that the repetition of variables will have an end in the process of the symmetrization of the non-symmetric functions. An intuitive proof is as follows: For totally symmetric functions the number of variables are equal to the number of levels of the lattice network, as there is no need to repeat variables, and as it is known that by the repetition of variables every non-symmetric function is symmetrized, then this must result in definite number of levels in the corresponding lattice network and as a consequence in certain number of total variables, repeated and non-repeated, that will result in the termination of the process of symmetrization. One method to define the symmetry of a Boolean function is by using symmetry indices $S^{i}$ [28].

Definition 2. A symmetry index $\left(S^{i}\right)$ has superscript $i$ equals to the count of the number of "1" values in the states of variables in the corresponding cell in a Karnaugh map.

Example 2 illustrates the concept of symmetry indices for a two-variable Boolean function, and shows the close relationship between two-dimensional lattice networks and symmetry indices.

Example 2. For the binary non-symmetric implication function $F=a^{\prime}+b$, Fig. 5 illustrates the relationship between the karnaugh map with non-conflicting symmetry indices $S^{i}$ and the twodimensional lattice network with non-conflicting leaves, which is achieved by repeating variable $\{a\}$ twice in the two-dimensional lattice network.

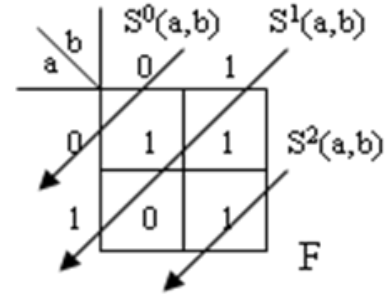

(a)

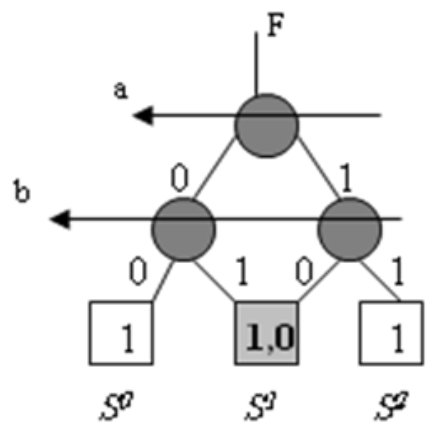

(c)

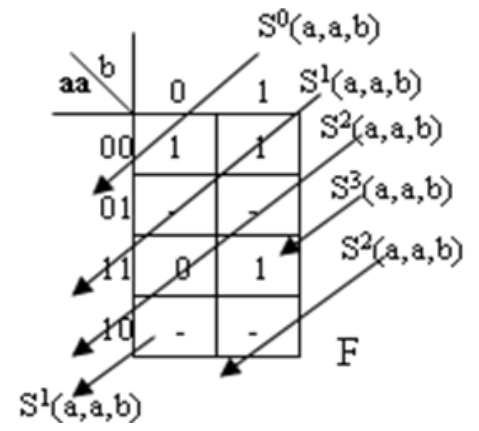

(b)

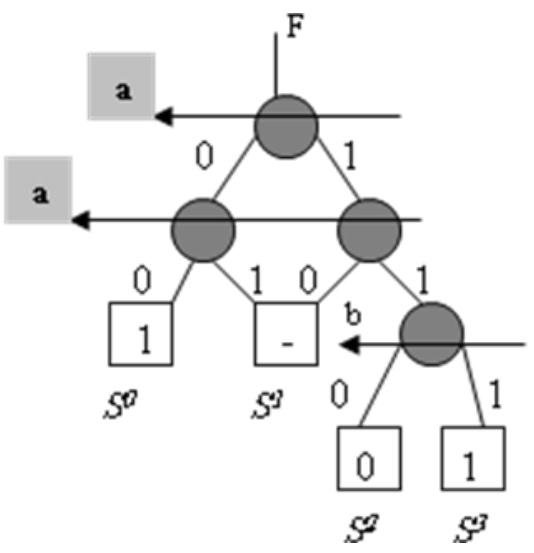

(d)

Figure 5. Using symmetry indices $S^{i}$ : (a) non-symmetric implication Boolean function, (b) symmetrization by the repetition of variable $\{a\}$, (c) two-dimensional lattice network that corresponds to Fig. 5(a) with conflicting leaf (in dark box), and (d) two-dimensional lattice network that corresponds to Fig. 5(b) with non-conflicting leaves. 
It is to be noted that all internal nodes in Fig. 5 are two-to-one multiplexers (i.e., selectors), where if one multiplies each leaf value (from left to right) with all possible bottom-up paths (from the leaves to the root $F$ ) and add them over Boolean algebra then one obtains the required function $F$ in the root.

\section{THREE-DIMENSIONAL LATTICE NETWORKS}

The concept of two-dimensional lattice networks that was presented in Section 2 can be generalized to include the case of three-dimensional lattice networks [2]-[3]. Since the most natural way to think about a binary lattice network is the two-dimensional four-neighbor lattice network such as the one shown in Fig. 3(a), one can extend the same idea to utilize the full threedimensional space in the case of ternary lattice networks. Such lattices represent threedimensional six-neighbor lattice networks.

Although regular lattice networks can be realized in the three-dimensional space for the third Galois radix while maintaining their full regularity, they are unrealizable for radices higher than three. Higher dimensionality lattice networks can be realized in three-dimensional space but on the expense of loosing the full regularity. This is because the circuit realization for the ternary case produces a regular circuit in three dimensions that is fully regular in terms of interconnects, where all interconnects are of the same length. Realizing the higher dimensionality lattice networks in lower dimensionality space is possible but with the expense of regularity in which the resulting lattice networks will not be fully regular due to the uneven lengths of the resulting interconnects between nodes.

As a topological concept, and as stated previously, lattice networks can be created for two, three, four or any higher Galois radices. However, because our physical space is three-dimensional, lattice networks as a geometrical concept, can be realized in a solid material with all interconnects between the cells are of the same length, only for radix two within two-dimensional space or radix three within three-dimensional space. It is thus interesting to observe that the characteristic geometric regularity of the lattice networks realization which is observed for binary and ternary functions will be no longer observable for quaternary or higher radix functions. Thus, the ternary lattice networks have a unique position as regular structures that can make the best use of threedimensional space.

Because three-dimensional lattice networks exist in a three-dimensional space, a geometrical reference of coordinate systems is needed in order to be systematic in the realizations of the corresponding logic circuits. Consequently, the right-hand rule of the Cartesian coordinate system is adopted. As will be noted in Example 3, each dimension in a three-dimensional lattice network corresponds to a value of the corresponding control variable; value zero of the control variable propagates along the $x$-axis, value one of the control variable propagates along the $y$-axis, and value two of the control variable propagates along the $z$-axis.

Example 3. This example shows the realization of ternary 3-digit full adder using threedimensional lattice networks. Multi-valued addition is performed in this example utilizing the modsum operator. Table 2 shows the modsum addition for ternary inputs. 
Table 2. Ternary modsum addition.

\begin{tabular}{|c|c|c|c|}
\hline & 0 & 1 & 2 \\
\hline 0 & 0 & 1 & 2 \\
\hline 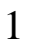 & 1 & 2 & 0 \\
\hline & 2 & 0 & 1 \\
\hline
\end{tabular}

Figure 6 shows the logic circuit of the ternary 3-digit full adder that implements the ternary addition operation presented in Table 2.

The following ternary maps in Fig. 7 represent the ternary Sum $(S)$ and the ternary output Carry $\left(C_{\text {out }}\right)$ functions that appear in the logic circuit in Fig. 6. The presented representation is performed by using 1-RPLs for variables $a, b$ and $c$, and using the corresponding Galois field addition and multiplication operations.

The lattice architectures that are shown in Figs. 8 and 9 show the corresponding threedimensional lattice realizations of the ternary maps in Fig. 7 which represent the Sum and output Carry ternary functions.

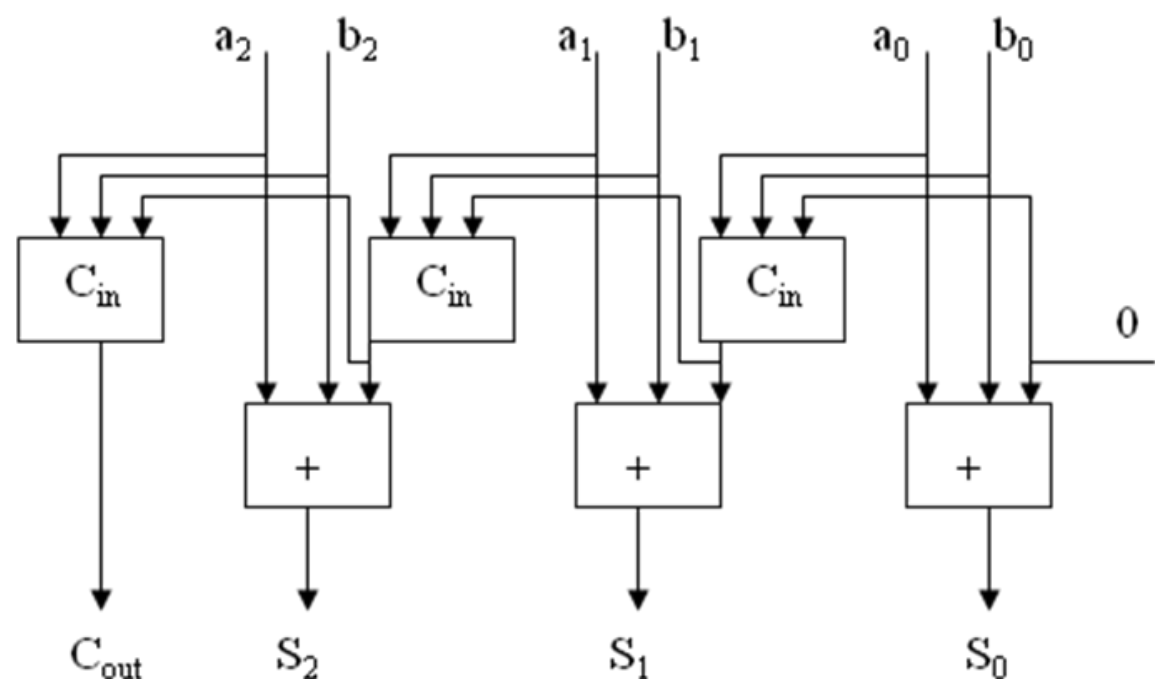

Figure 6. Logic circuit representation of a ternary 3-digit full adder. 


\begin{tabular}{|c|c|c|}
\hline$a b$ & 0 & 1 \\
\hline $\begin{array}{ll}0 & 0\end{array}$ & 0 & 1 \\
\hline $\begin{array}{ll}0 & 1\end{array}$ & 1 & 2 \\
\hline 02 & 2 & 0 \\
\hline 10 & 1 & 2 \\
\hline 11 & 2 & 0 \\
\hline 12 & 0 & 1 \\
\hline 20 & 2 & 0 \\
\hline 21 & 0 & 1 \\
\hline 22 & 1 & 2 \\
\hline
\end{tabular}

(a)

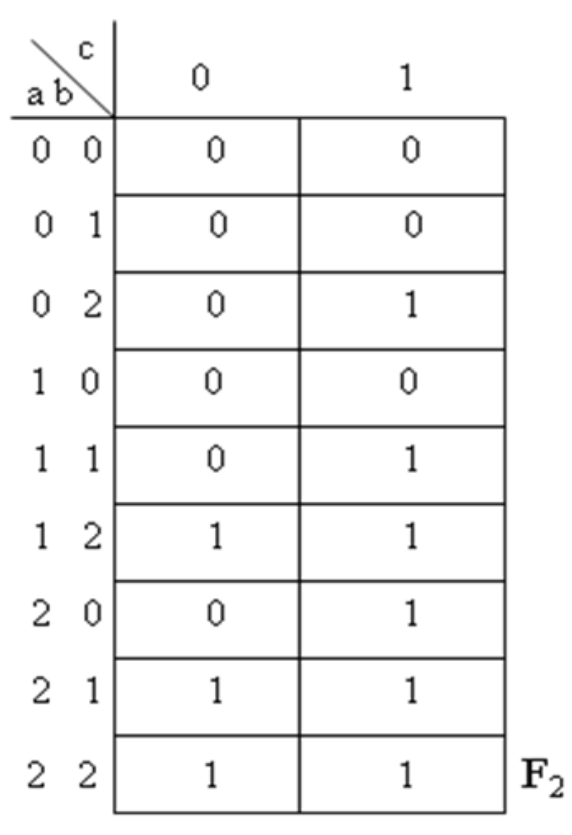

(b)

Figure 7. Ternary maps for (a) ternary Sum: $F_{1}={ }^{0} a^{0} b^{1} c+{ }^{0} a^{1} b^{0} c+2 \cdot{ }^{0} a^{1} b^{1} c+2 \cdot{ }^{0} a^{2} b^{0} c+{ }^{1} a^{0} b^{0} c+2 \cdot{ }^{1} a^{0} b^{1} c+$ $2 \cdot{ }^{1} a^{1} b^{0} c+{ }^{1} a^{2} b^{1} c+2 \cdot{ }^{2} a^{0} b^{0} c+{ }^{2} a^{1} b^{1} c+{ }^{2} a^{2} b^{0} c+2 \cdot{ }^{2} a^{2} b^{1} c$, and (b) ternary Carry: $F_{2}={ }^{0} a^{2} b^{1} c+{ }^{1} a^{1} b^{1} c+{ }^{1} a^{2} b^{0} c$ $+{ }^{1} a^{2} b^{1} c+{ }^{2} a^{0} b^{1} c+{ }^{2} a^{1} b^{0} c+{ }^{2} a^{1} b^{1} c+{ }^{2} a^{2} b^{0} c+{ }^{2} a^{2} b^{1} c$.

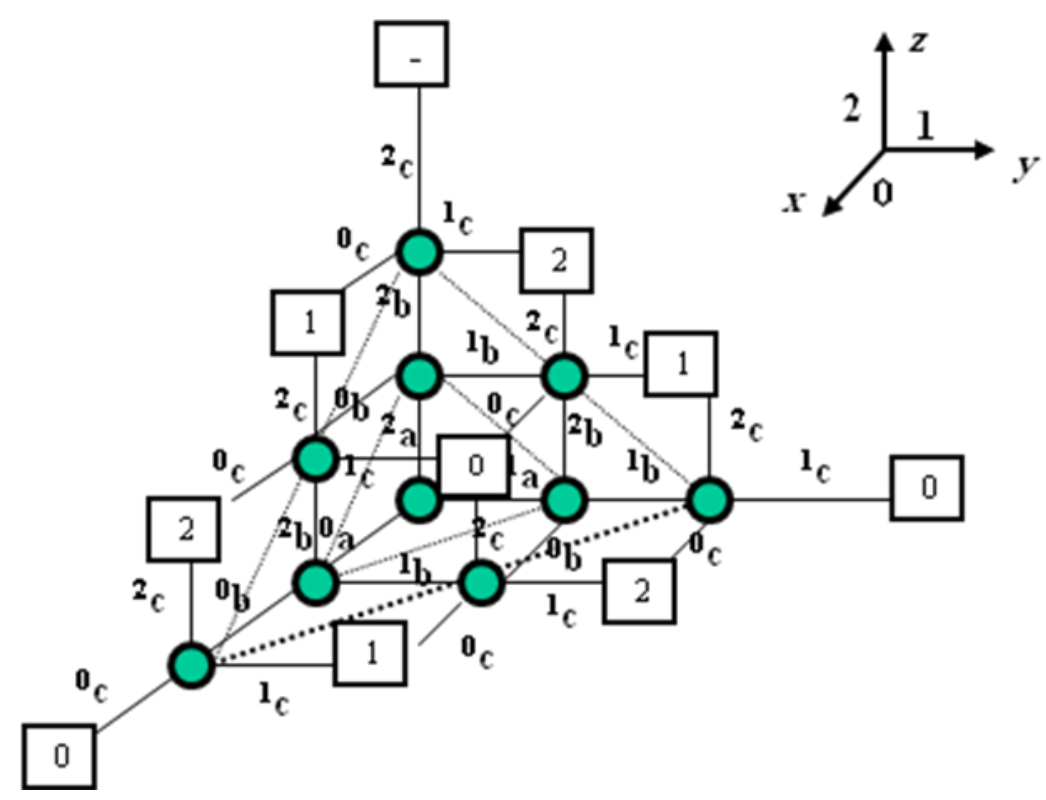

Figure 8 . The Sum function $F_{1}$ of the ternary 3 -digit full adder, where - is a ternary don't care $(0,1$, or 2$)$. 


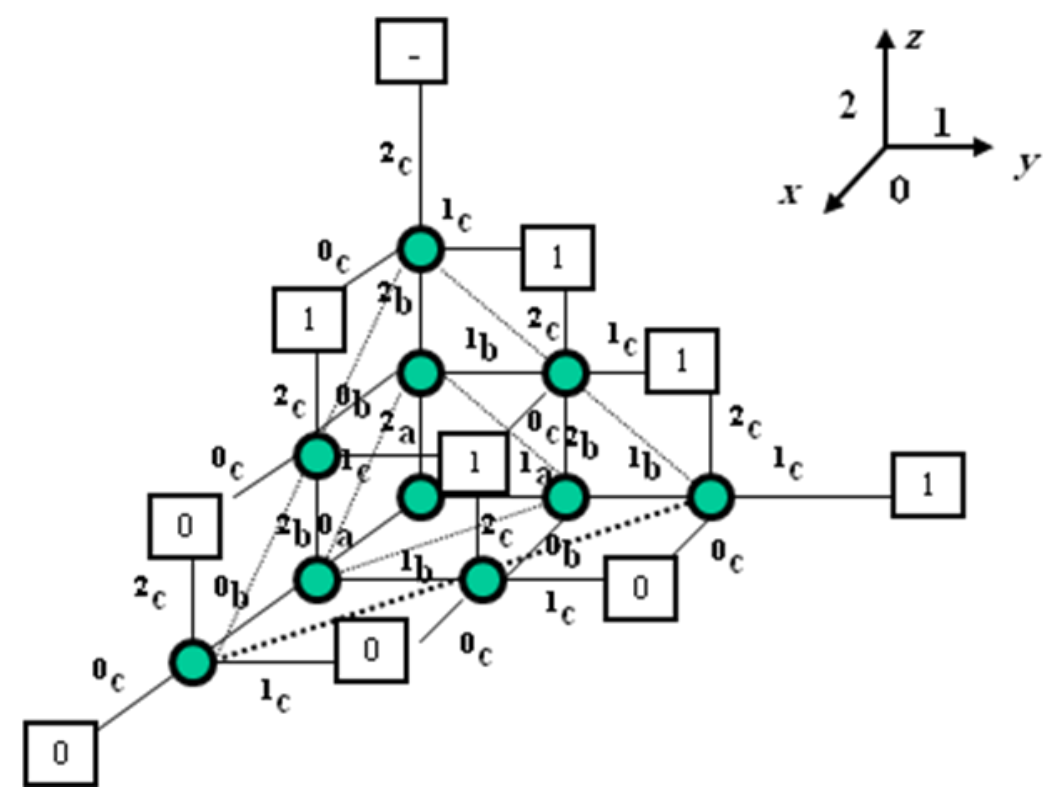

Figure 9. The Carry function $F_{2}$ of the ternary 3-digit full adder, where - is a ternary don't care $(0,1$, or 2$)$.

As observed in Example 3, Figs. 8 and 9 represent a fully regular lattice network in threedimensions. Each dimension corresponds to a value of the corresponding control variable: value zero of the control variable propagates along the $x$-axis, value one of the control variable propagates along the $y$-axis, and value two of the control variable propagates along the $z$-axis. Since the ternary function in Example 3 is fully symmetric, no variables are needed to be repeated in the corresponding lattice network. In three-dimensional space, each control variable spreads in a plane to control the corresponding nodes (these parallel planes are represented using the corresponding dotted triangles in Figs. 8 and 9), in contrast to the binary case where each control variable spreads in a line to control the corresponding nodes (these control signals are represented in the corresponding horizontal lines in Fig. 3(a)). Each node in Figs. 8 and 9 represents a threeinput one-output multiplexer whose output goes in three directions.

All internal nodes in Figs. 8 and 9 are three-to-one multiplexers (i.e., selectors), where if one multiplies each leaf value, going counter clock wise, with all possible out-to-in paths (from the leaves to the root) and add them over Galois field (in Table 1) then one obtains the maps in Fig. $7\left(\right.$ a) $\left(F_{1}\right)$ and Fig. $7\left(\right.$ b) $\left(F_{2}\right)$, respectively. Also, one notes that internal nodes in Figs. 8 and 9 lay on the corners of three-dimensional cubes, which is in contrast to the binary case (e.g., Fig. 4) where nodes lay on the corners of two-dimensional squares. From Figs. 8 and 9, one observes that the Sum and Carry functions are both symmetric, since there is no conflict in leaf values, and consequently there is no need to repeat variables to make the ternary functions realizable in the corresponding three-dimensional lattice networks. In ternary non-symmetric functions, at least one leaf has conflict values, and one needs to repeat variables to symmetrize the corresponding non-symmetric functions, in order to realize such functions in the corresponding threedimensional lattice networks. Example 4 shows the realization of a ternary non-symmetric function in a three-dimensional lattice through the process of repeating variables.

Example 4. For the non-symmetric two-variable ternary-input ternary-output function $F=a b+$ $a^{\prime} b^{\prime}$ ', and by adopting the right-hand rule of the Cartesian coordinate system, Fig. 10 illustrates the three-dimensional logic network to implement such non-symmetric function. In Figs. 10(c) and 10(d), if one multiplies each leaf value, going counter clock wise, with all possible out-to-in paths (from the leaves to the root) and add them over Galois field (in Table 1), then one obtains 
the maps in Figs. 10(a) and 10(b), respectively, where $\left\{{ }^{0} a,{ }^{1} a,{ }^{2} a\right\}$ are the zero, first and second polarities of the 1-RPL of variable $a,\left\{{ }^{0} b,{ }^{1} b,{ }^{2} b\right\}$ are the zero, first and second polarities of the 1RPL of variable $b$, and ternary-valued variables $a$ and $b$ can take any value in the set $\{0,1,2\}$.

\section{CARbON NANOTUBES AND NANOTIPS}

This section presents important background on carbon-based nanotubes and nanotips and their corresponding characteristics. The presented carbon-based nanotubes and nanotips will be utilized in building controlled-switching that will be used in the second and third parts of the article for the construction of nano three-dimensional lattice networks.

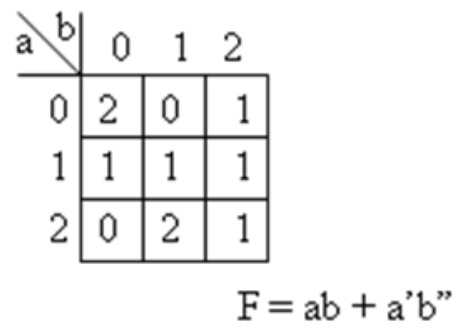

(a)

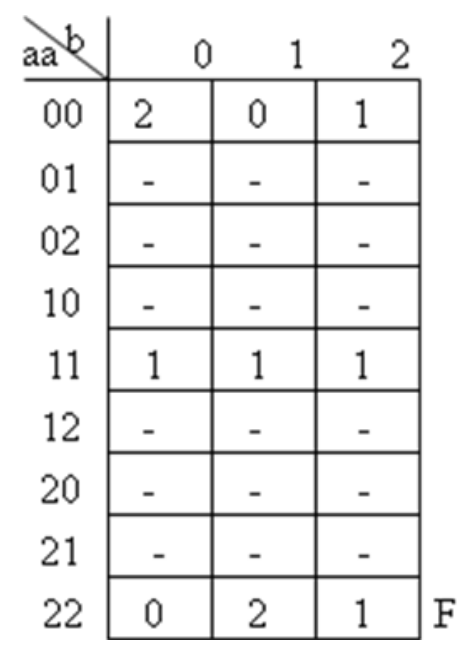

(b)

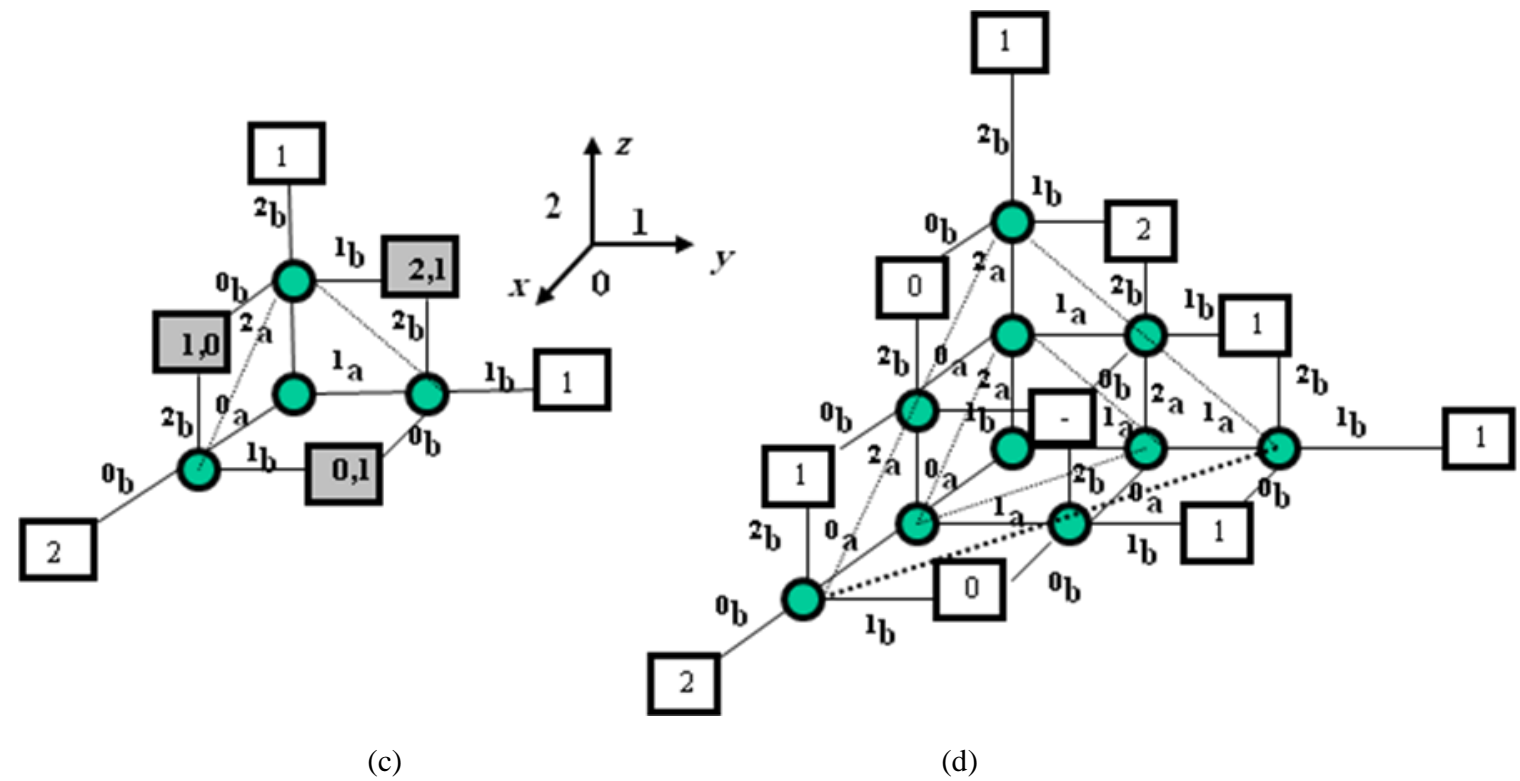

Figure 10. Three-dimensional lattice networks for non-symmetric functions: (a) Ternary non-symmetric function, (b) symmetrization by repeating variable $a$, (c) three-dimensional lattice network that corresponds to Fig. 10(a) with conflicting leaves (in dark boxes), and (d) final three-dimensional lattice network that corresponds to Fig. 10(b) with non-conflicting leaves. 


\subsection{Carbon Nanotubes Characteristics and Properties}

The CNT, which is a cylindrical sheet of graphite, is formed geometrically in two distinct forms which affect CNT properties: (a) straight CNT in which a CNT is formed as a straight cut from graphite sheet and rolled into a carbon nanotube and (b) twisted CNT in which a CNT is formed as a cut at an angle from graphite sheet and rolled into a carbon nanotube.

The newly emerging CNT technology has been implemented in many new promising applications [4]- [7], [10]-[11], [15]-[19], [22], [26], [29], [34]. This includes TVs that are based on the field emission of CNTs that consume much less power, thinner and have much higher resolution than the best plasma-based TVs available, nano circuits based on CNTs such as CNT-based FETs that consume less power and are much faster than the available silicon-based FETs, carbon nanocoils that can be used as inductors in nanofilters and as nanosprings in nano dynamic systems, and CNT rings. In addition, the CNT has also been demonstrated for potential exciting applications such as in CNT probes, new composite materials, CNT data storage devices capable of storing $10^{15}$ bytes $/ \mathrm{cm}^{2}$, drug delivery systems, nano lithography, and CNT gears in which larger gears drive smaller gears or vice versa The CNT growth, as observed using Transmission Electron Microscopy (TEM), Atomic Force Microscopy (AFM) and Scanning Electron Microscopy (SEM), requires processes with correct conditions and materials. Currently, several methods for growing various types of CNTs exist which include: A big spark between two graphite rods, few millimeters apart, that are wired to a power supply in which a $10^{2}$ Ampere spark between the two rods vaporizes carbon into hot plasma which partially re-condenses into the form of CNT, chemical vapor deposition (CVD) of a hot gas such as methane in which a substrate is placed in an oven then the oven is heated to approximately 600 degrees Celsius and slowly methane is added; as methane decomposes, it frees carbon atoms that partially re-compose into the form of 0.6-1.2 nm in diameter SWCNTs, and a laser blast of a graphite target in which laser pulses blasts a graphite rod which generates hot carbon gas from which CNT forms.

Table 3 summarizes most of CNT properties as compared to traditional counterparts [4]. For example, the field emission property is used in the recently developed highly efficient CNT-based TVs and is used in newly developed prototype vacuum tube lamps in six colors that are twice as bright as conventional light bulbs, longer-lived and at least ten times more energy-efficient. The properties of current carrying capacity, thermal stability, power consumption and electron scattering are qualifying the CNT for very promising future use in highly efficient power transmission. The property of preserving the electron spin will be utilized in using the CNT for reliable quantum computing. The size property is very useful for using CNTs as nanowires that would result in decreasing the total size of areas and volumes that are occupied by wires and interconnects in the corresponding integrated circuits. The property of resilience is useful in building circuits and structures that has to maintain stress without structural damages. The CNT property of energy band gaps qualifies CNTs to be used in wide applications that require wide range of energy band gaps from the conductor state to the semiconductor state.

In addition, CNTs are excellent field emitters and facilitate the miniaturization of electronic devices. Furthermore, the kinetic inductance of CNT causes them to be high impedance (of approximately $5 \mathrm{k} \Omega$ ) transmission lines, and had shown that this effect can be used for efficient broadband matching to the high impedance that is inherent in field emission. The static and dynamic characterizations of field emitters consisting of a single CNT (both single-walled and multi-walled) were described [24] and the comparison to a field emitter consisting of an etched single crystal of tungsten was also performed. 
Table 3. Important properties and charateristics of carbon nanotubes.

\begin{tabular}{|c|c|c|}
\hline Property & Single-Walled CNT & By Comparison \\
\hline Size & $0.6-1.8 \mathrm{~nm}$ in diameter & $\begin{array}{l}\text { Electron beam lithography } \\
\text { can create lines } 50 \mathrm{~nm} \\
\text { wide, and a few nm thick }\end{array}$ \\
\hline Density & $1.33-1.40 \mathrm{~g} / \mathrm{cm}^{3}$ & $\begin{array}{c}\text { Aluminum has a density of } \\
2.7 \mathrm{~g} / \mathrm{cm}^{3}\end{array}$ \\
\hline Tensile Strength & $\approx 45 \cdot 10^{9}$ Pascals & $\begin{array}{l}\text { High-strength steel alloys } \\
\text { break at } \approx 2 \cdot 10^{9} \text { Pascals }\end{array}$ \\
\hline Resilience & $\begin{array}{c}\text { Can be bent at large angles } \\
\text { and re-straightened } \\
\text { without damage }\end{array}$ & $\begin{array}{l}\text { Metals and carbon fibers } \\
\text { fracture at grain boundaries }\end{array}$ \\
\hline Current Carrying Capacity & $\approx 1 \cdot 10^{9} \mathrm{~A} / \mathrm{cm}^{2}$ & $\begin{array}{l}\text { Copper wires burn out at } \\
\approx 1 \cdot 10^{6} \mathrm{~A} / \mathrm{cm}^{2}\end{array}$ \\
\hline Field Emission & $\begin{array}{c}\text { Can activate phosphors at } \\
1-3 \mathrm{~V} \text { if electrodes are } \\
\text { spaced } 1 \text { micron apart }\end{array}$ & $\begin{array}{l}\text { Molybdenum tips require } \\
\approx 50-100 \mathrm{~V} / \text { micrometer } \\
\text { with very limited lifetimes }\end{array}$ \\
\hline Heat Transmission & $\begin{array}{c}\approx 6,000 \mathrm{~W} / \mathrm{m} \cdot \mathrm{K} \text { at room } \\
\text { temperature }\end{array}$ & $\begin{array}{c}\text { Nearly pure diamond } \\
\text { transmits } \approx 3,320 \mathrm{~W} / \mathrm{m} \cdot \mathrm{K}\end{array}$ \\
\hline $\begin{array}{c}\text { Temperature/Thermal } \\
\text { Stability }\end{array}$ & $\begin{array}{l}\text { Stable up to } 2,800 \mathrm{C} \text { in } \\
\text { vacuum, and } 750 \mathrm{C} \text { in air }\end{array}$ & $\begin{array}{l}\text { Metal wires in microchips } \\
\text { melt at } \approx 600-1,000 \mathrm{C}\end{array}$ \\
\hline Cost & $\approx 1,500 \$ / \mathrm{g}$ & Gold sells for $\approx 10 \$ / \mathrm{g}$ \\
\hline $\begin{array}{l}\text { Preservation of the Quantum } \\
\text { Property of Electron Spin }\end{array}$ & Optimal; Very High & Low in regular conductors \\
\hline Power Consumption & Very low & Higher in metal wires \\
\hline Speed & $\begin{array}{c}\text { Very high; } \geq 1 \cdot 10^{12} \text { Hertz } \\
\text { nanoscale switch }\end{array}$ & $\begin{array}{c}\geq 1,000 \text { times as fast as } \\
\text { processors available today }\end{array}$ \\
\hline $\begin{array}{c}\text { Electron Scattering } \\
\text { (Resistance) }\end{array}$ & Almost none & Comparatively high \\
\hline Energy Band Gaps & $\begin{array}{l}\text { Easily tunable; Depends on } \\
\text { CNT diameter, and thus } \\
\text { wide range of band gaps } \\
\text { can be obtained; } \approx 0 \text { (like a } \\
\text { metal), as high as band gap } \\
\text { of Silicon, and almost } \\
\text { anywhere in between }\end{array}$ & $\begin{array}{l}\text { No other known material } \\
\text { can be so easily tuned }\end{array}$ \\
\hline
\end{tabular}

\subsection{Carbon Nanotube-Based Multiplexing Devices}

Figure 11 shows the synthesis of a logic inverter, that operates the same way as a CMOS inverter, using two nanotube FETs [18]. In Fig. 11, silicon ( $\mathrm{Si}$ ) is used as back-gate in the device, silicon dioxide $\left(\mathrm{SiO}_{2}\right)$ is used as an insulator, gold is used as electrodes (conductors), and PMMA is a cover that protects anything beneath it from being exposed to oxygen $\left(\mathrm{O}_{2}\right)$ where oxygen is used to convert n-CNTFET to $\mathrm{p}$-CNTFET. 


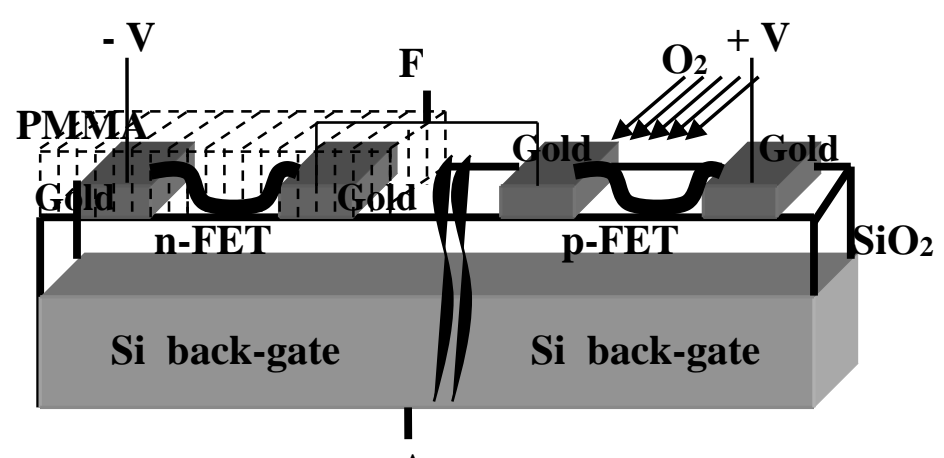

A

Figure 11. The NOT (inverter) logic gate using two nanotube-based FETs.

Figure 12 shows solid-state CNT-based multiplexer [4], [6] where CNT is used as a channel in a Field Effect Transistor (FET). As was shown in Fig. 11, the use of CNT as a channel in FET has been shown and a simple inverter that works exactly the same way as an ordinary CMOS inverter was built. Here, we use the same principle of using CNT as a channel but in a different device that uses two CNT n-FETs and two CNT p-FETs with different topologies of interconnects that are used for inputs $A$ and $B$, controls $C$ and $C^{\prime}$, and the output $F$. In Fig. 12, silicon ( $\mathrm{Si}$ ) is used as back-gate in the device, silicon dioxide $\left(\mathrm{SiO}_{2}\right)$ is used as an insulator, and gold is used as electrodes (conductors). Four metallic catalyst islands with each pair located at the facing ends of each pair of gold electrodes can be also used to grow CNTs between each pair of gold electrodes, rather than just placing CNTs in contact with the gold electrodes. The PMMA is a cover that protects anything beneath it from being exposed to oxygen $\left(\mathrm{O}_{2}\right)$ where oxygen is used to convert an n-CNTFET to a p-CNTFET.

The fabrication of each sub-device in Fig. 12 is done as follows [18]: Initially the two CNTFETs are p-type. After vacuum annealing both CNTFETs are converted to n-type. The two CNTFETs are exposed to oxygen $\left(10^{-3}\right.$ Torr of oxygen for three minutes), and the unprotected n-CNTFET converts back to the original p-type, while the protected CNTFET remains n-type. Another method to form p-type and n-type CNTFETs has been reported as follows: CNT channel dopped with potassium $(\mathrm{K})$ produces an n-type CNTFET, while a CNT without dopping produces p-type CNTFET. Dopping produces n-type CNTFET by shifting the Fermi energy level to the conduction band that results in an increase of electron concentration in the conduction band which increases the conductance of the FET for a given positive gate voltage.

The function of the device in Fig. 12 can be analyzed as follows [6]: If $C=$ "1" then the upper transmission gate (t-gate) is activated and $A$ is passed to $F$ while the lower t-gate is deactivated and $B$ is not passed to $F$, and if $C=$ " 0 " then the lower t-gate is activated and $B$ is passed to $F$ while the upper t-gate is deactivated and $A$ is not passed to $F$. Thus, the solid-state CNT multiplexer functions exactly as a 2-to-1 multiplexer. Intra-molecular CNTFET technology [18] can be used instead of each sub-device in Fig. 12 in which a single CNT bundle is placed on top of three gold electrodes to produce an n-type and a p-type CNTFETs on the same substrate using the exact procedure that is used for the inter-molecular CNTFET in Fig. 12.

Figure 13 shows the magnetic CNT-based multiplexer [4], [6]. The numbers on the parts of the device in Fig. 13 indicate the following: (\#1, \#2, \#3, \#4, \#5, \#6) are CNTs, (\#7, \#8, \#9, \#10, \#11) are electrical current directions, (\#12) is the body of the device which is an electrical insulator such as glass, $\mathrm{SiO}_{2}$, plastics or any other type of electrical insulator, and (\#13) is a hollow space with structural walls in two opposite sides (sides of CNTs \#1 and \#2) made of an electrical insulator. 


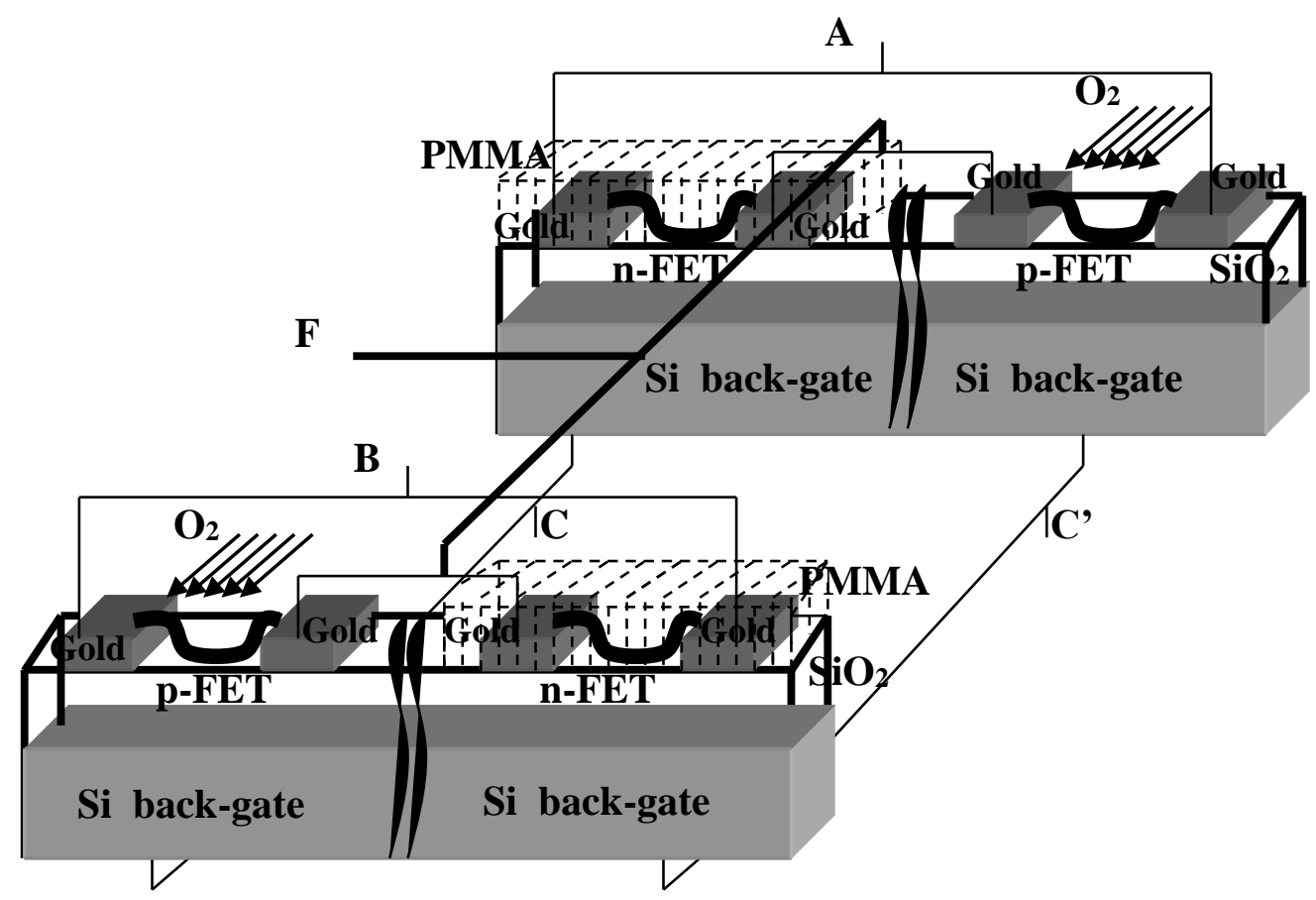

Figure 12. The device structure of solid-state inter-molecular CNT multiplexer.

The device in Fig. 13 operates as follows: electrical current (\#7) is input $B$ and can have two levels $x$ and $y$ that indicate logics " 0 " and " 1 ", respectively. Electrical current (\#8) is input $A$ and can have two levels $x$ and $y$ that indicate logics " 0 " and "1", respectively. The two electrical currents (\#7, \#8) are flowing in the directions indicated in Fig. 13 in CNTs (\#1) and (\#2), respectively. The flow of these currents will produce magnetic fields around CNTs (\#1, \#2) according to Ampere's (circuital) law in the Maxwell's equations [33] that relates the magnetic field around a closed loop to the electric current passing through the loop, for which the differential form representation in terms of the total charge and current takes the formulation as shown in Equation (5).

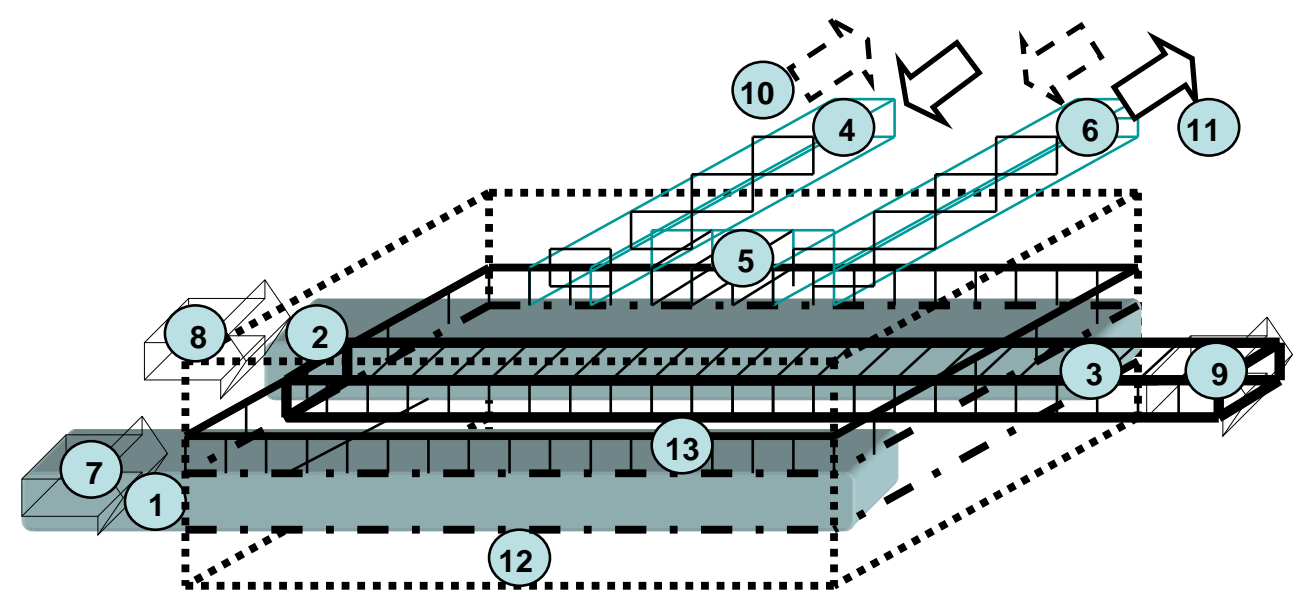

Figure 13. The device structure of the magnetic CNT-based multiplexer. 


$$
\vec{\nabla} \times \vec{B}=\mu_{0} \vec{J}+\varepsilon_{0} \mu_{0} \frac{\partial \vec{E}}{\partial t}
$$

where $\vec{B}=\mu \vec{H}$ is the magnetic field (magnetic flux density; magnetic field density), $\vec{H}$ is the magnetizing field (or magnetic field intensity), $\vec{\nabla} \times \vec{B}$ is the curl of the magnetic field, $\mu_{0}$ is permeability, $\varepsilon_{0}$ is permittivity, $\vec{J}$ is current density, $\vec{E}=\frac{\vec{D}}{\varepsilon}$ is the electric field, $\vec{D}$ is the electric displacement field (or electric flux density), and $\frac{\partial \vec{E}}{\partial t}$ is the time derivative of the electric field. The direction of such magnetic fields follows the conventional right-hand thumb rule [33].

The CNT (\#3) initially is in contact with either CNT (\#1) or CNT (\#2), and thus a current would be running in CNT (\#3) in the same direction as currents (\#7, \#8). An electrical current is pumped through CNTs (\#4, \#5, \#6), and this current can be pumped either in clock wise (CW) direction (\#10) or counter clock wise (CCW) direction (\#11). The electrical current (\#10) flowing in CNTs (\#4, \#5, \#6) will produce a magnetic field according to Maxwell's equations in a direction according to the right-hand thumb rule, and if current (\#11) flows in CNTs (\#4, \#5, \#6) a magnetic field will be produced in an opposite direction of that which is produced by current (\#10). The direction of the current flowing in CNTs (\#4, \#5, \#6) and thus the direction of the magnetic fields plays the role of the control signal in a regular multiplexer as follows: if current (\#11) is flowing in CNTs (\#4, \#5, \#6) then an attractive Lorentz force occurs [33]:

$$
\vec{F}=\vec{I} \times \vec{B}
$$

where $\vec{I} \times \vec{B}$ is the cross product between current $\vec{I}$ in a conductor that lays in magnetic field $\vec{B}$, and $\vec{F}$ is the Lorentz force between two current-carrying conductors that occurs between CNT (\#5) and CNT (\#3) that causes CNT (\#3) to move in the space (\#13) towards CNT (\#5) and thus makes a contact with CNT (\#2) which means that current (\#8) or input $A$ is selected to flow to the output. On the other hand, if current (\#10) is flowing in CNTs (\#4, \#5, \#6) then a repulsive Lorentz force occurs between CNT (\#5) and CNT (\#3) that causes CNT (\#3) to move in the space (\#13) away from CNT (\#5) and thus makes a contact with CNT (\#1) which means that current (\#7) or input $B$ is selected to flow to the output. Thus, the nano mechanical device in Fig. 13 implements a two-to-one logic multiplexer (selector). It has to be noted that one has to wait a period of time equal to $T$ in order to obtain the result at the output. This time indicates the traveling (or displacement) time needed for CNT (\#3) to make a contact with either CNT (\#1) or CNT (\#2) depending on the direction of the electrical current flowing in CNTs (\#4, \#5, \#6).

\subsection{Carbon Nanotubes-BaSed Field Emitters}

Field electron emission is the emission of electrons from the surface of a cathode under the influence of the applied electric field (of $3 \times 10^{9} \mathrm{~V} / \mathrm{m}$ ) which is strongly dependent upon the work function of the emitting material. The general form of Fowler-Nordheim-type (FN-type) equation [21] can be produced as follows [20]:

$$
J=\lambda_{L} a \varphi^{-1} F^{2} E X P\left[-V_{F} b \varphi^{3 / 2} / F\right]
$$

Equation (7) is used in all cases of field emission processes, where $J$ is the local emission current density, $a$ and $b$ are the first and the second Fowler - Nordheim constants, respectively, $v_{\mathrm{F}}$ is the barrier form correction factor and it accounts for the particular shape of the potential barrier 
model, and $\lambda_{\mathrm{L}}$ is the local pre-exponential correction factor where it takes into account all of the other factors that influence the emission. The factors $v_{\mathrm{F}}$ and $\lambda_{\mathrm{L}}$ depend on the applied field $F$.

This section presents important functional modeling of field emitters and the corresponding experimental setups and measurements that characterize their time-dependent response. In order to perform the static modeling of CNT field emitters, four field emission carbon nanotubes, which are shown in Figs. 14(b)-14(e), were manufactured by Xintek, Inc. The copper anode is at the right and the CNT emitter is mounted on a tungsten wire attached to the copper cylinder at the left as shown in Fig. 14(a). Figures 14(b)-14(e) show the images of CNT emitters for each carbon nanotube, taken with a JEOL Ltd. model JEM 6300 SEM [24]. Carbon nanotubes M-1 and M-4 have a single MWCNT as the emitter, and carbon nanotubes C-3 and C-6 have a single SWCNT as the emitter. The used CNTs were formed in bundles that have diameters of 10-30 nm, but in each carbon nanotube the field emission is from the one CNT at the end of the bundle where the electric field is most intense.

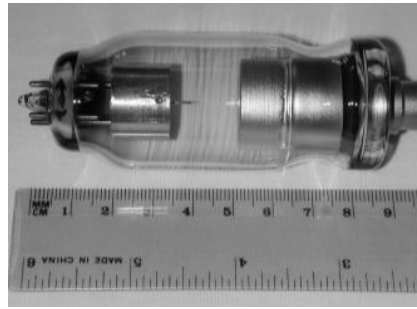

(a)

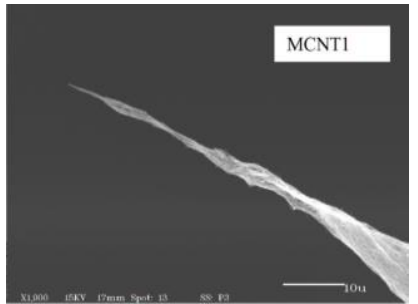

(b)

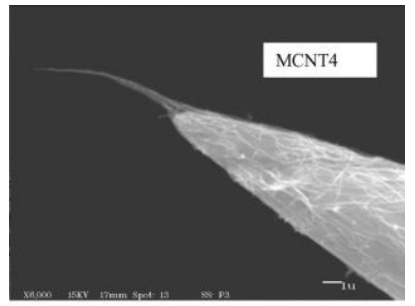

(c)

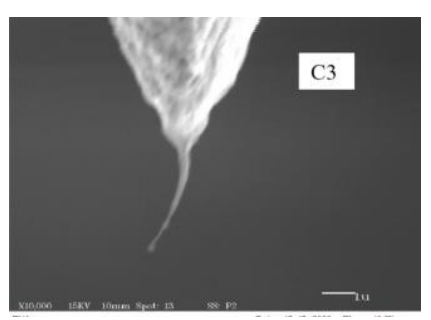

(d)

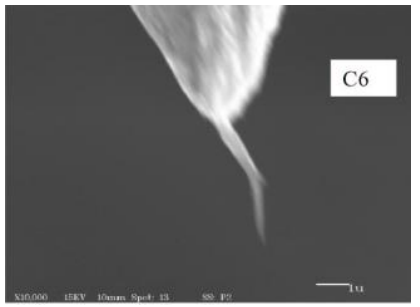

(e)

Figure 14. Carbon nanotube - based field emission: (a) structure of the field emission carbon nanotubes manufactured by Xintek Inc. and (b) - (e) Scanning Electron Microscopy (SEM) images of the CNT emitters in the four utilized carbon nanotubes.

The DC current-voltage characteristics were measured for these four carbon nanotubes, as well as a field emitter tube from Leybold Didactic $\mathrm{GmbH}$, which has an etched single crystal of tungsten as the emitter. All of the measurements that were made with the five tubes were performed at room temperature. The tungsten tip is mounted on a filament so that this tip is heated for cleaning shortly before each session of measurements. However, it is not possible to clean the CNT, which probably causes the "switch-on" effect in which the supply voltage must be momentarily increased well beyond the operating point to initiate field emission with the CNT. The data from the DC measurements were reduced by the Fowler-Nordheim analysis which is based on the following simplified form of the Fowler-Nordheim equation that provides the magnitude of the current density as a function of the applied static field for the field emission from a specific material:

$$
J=A E^{2} \mathrm{e}^{(-B / E)}
$$

where $J$ and $E$ are the magnitudes of the current density and the electric field intensity, $A=1.541$ x $10^{-6} / \Phi$, and $B=6.831 \times 10^{9} \Phi^{3 / 2}$. The work function values are $\Phi=4.5 \mathrm{eV}$ for tungsten and (for 
the CNT) was set to $\Phi=4.9 \mathrm{eV}$ for graphene. In order to apply the Fowler-Nordheim equation to the DC current-voltage data, the following equation was also used which is valid for a given carbon nanotube, where $I$ is the field emission current and $V$ is the potential applied between the anode and the cathode:

$$
I=C V^{2} \mathrm{e}^{(-D / V)}
$$

Equations (8) and (9) can be combined to obtain the following equations for the parameters $S$ and $R$, which are used to characterize the field emitters:

$$
\begin{aligned}
& S=C D^{2} / A B^{2} \\
& R \equiv V / E=D / B
\end{aligned}
$$

where parameter $S$ refers to the effective emitting area which would be the physical area of the emitter if the current density were uniform over a fixed area and zero elsewhere, and parameter $R$ refers to the effective radius of curvature of the emitter but also includes the effects of local intensification of the electric field caused by elongation of the emitter or the reduction of the field which may be caused by shielding due to adjacent structures.

The Fowler-Nordheim plots of the DC current-voltage data were conducted using $\ln \left(I / V^{2}\right)$ as the ordinate and $(1 / V)$ as the abscissa. Equation (9) requires that these plots should be straight lines and typically with correlation $c \approx-0.998$. Linear regressions based on these Fowler-Nordheim plots typically have a standard variance $\sigma \approx 0.08$, and the probability for the null-hypothesis, that no linear relationship exists, is less than 0.0001 . The values of the parameters $\{C, D, S, R\}$ were determined from the linear regressions. A series ballast resistor of $100 \mathrm{M} \Omega$ was typically used in the measurements. However, when the series ballast resistor was increased to $2.575 \mathrm{G} \Omega$ with carbon nanotube C-6, the obtained data were not consistent with the Fowler-Nordheim equation ( $c=-0.846, \sigma=0.738)$ even though the emitted current was stable at each value of the applied static potential. Figure 15 shows the anomalous data which were obtained using the $2.575 \mathrm{G} \Omega$ ballast resistor. In order to explain this effect, it is hypothesized that for currents greater than 500 $\mathrm{nA}$, field emission with a single CNT may be intermittent, fluctuating at a high frequency. Thus, the average current, as measured by the DC microammeter, may be much greater with a large ballast resistor and this is because at those times when the current is momentarily low, the voltage drop across the ballast resistor is at minimum so that high voltage occurs across the CNT, and this voltage causes a short-duration surge in the current.

The values of parameter $R$, the effective radius of curvature of the emitter, were found to vary within 77-110 nm for the four carbon nanotubes with CNT emitters. This suggests that values of the local electric field at the emitting sites were as high as $14 \mathrm{~V} / \mathrm{nm}$ in some of these measurements. Others studying the field emission from various CNTs have provided approximate values for the electric field by dividing the applied voltage by the distance between the anode and the emitting tip, noting that this field would be intensified by the shape of the CNT, but not estimating the local electric field at the emitting sites [30]. The Fowler-Nordheim analysis gave a value of $91 \mathrm{~nm}$ for the effective radius of curvature of the emitter in the Leybold tube, suggesting that the local electric field was as high as $5 \mathrm{~V} / \mathrm{nm}$ in some of the performed measurements [24]. Current densities as high as $10^{9}$ and $10^{12} \mathrm{~A} / \mathrm{m}^{2}$ may be drawn from a tungsten emitter in steadystate and pulsed operation, respectively, and the corresponding values of the applied static field are 4.7 and $8.6 \mathrm{~V} / \mathrm{nm}$ which may be considered as limiting field strengths for tungsten under these conditions. Thus, the value of the parameter $R$ which was obtained for the Leybold tube appears to be reasonable.

The Fowler-Nordheim analysis also showed that the parameter $S$, the effective emitting area, varied within $81-230 \mathrm{~nm}^{2}$ for the four carbon nanotubes with CNT emitters. If the current density was uniform, this would correspond to circular emitting spots having radii of approximately 5-9 
nm. Others have used Lorenz microscopy to directly observe the emitting sites for field emission from MWCNT, and they found one or more sites having radii of several nm, and their data were in reasonable agreement with other performed experiments.

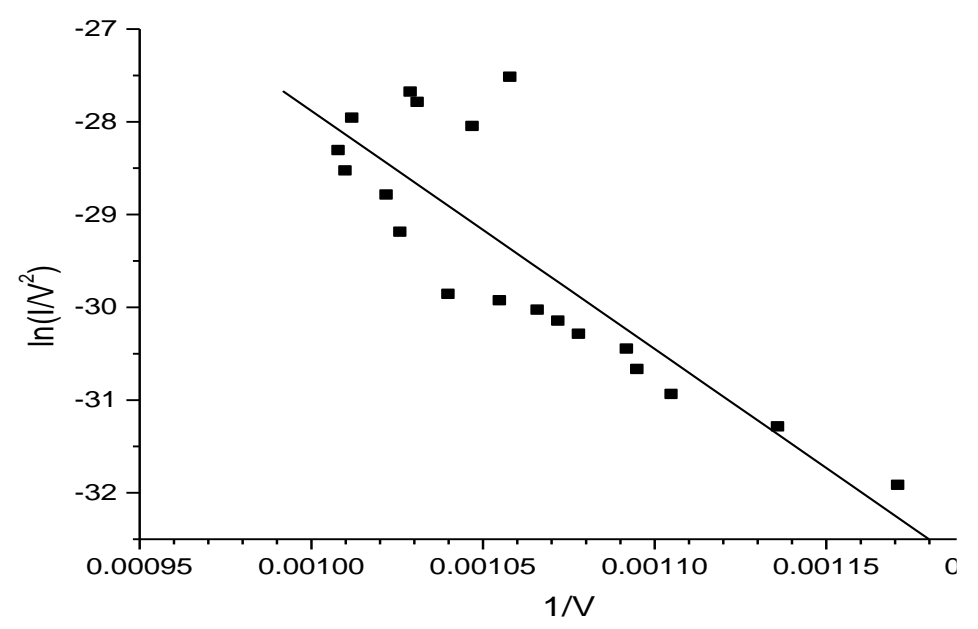

Figure 15. The obtained Fowler-Nordheim plot for CNT C-6 with 2.575 G $\Omega$ ballast resistor.

The Fowler-Nordheim analysis also showed that the effective emitting area for the tungsten tip in the Leybold tube would correspond to a hemisphere with a radius of $290 \mathrm{~nm}$. This result and the value of $91 \mathrm{~nm}$ for the effective radius of curvature of the emitter in the Leybold tube were in reasonable agreement with the radius of 100-200 $\mathrm{nm}$ which was specified by Leybold.

Recently conducted simulations and experiments had shown that photomixing (i.e., optical heterodyning) in laser-assisted field emission could be used as a new microwave or terahertz (THz) source with a multi-octave bandwidth [9], [12], [14], [25]. The field emitter tip used is much smaller than the wavelength of the incident optical radiation so quasi-static conditions require that the electric field of the radiation is superimposed on the applied static field to modulate the height of the energy barrier. Electrons tunnel from the tip into vacuum with a delay $\tau$ of less than 2 fs. Therefore, because the current-voltage characteristics of field emission are extremely nonlinear, it is shown that if two lasers are focused on the tip, the mixer current would follow each cycle of the difference frequency of the two lasers from DC up to $500 \mathrm{THz}$ (which is equal to $1 / \tau)$. It is also shown that the tip will withstand applied static fields as high as $9 \mathrm{~V} / \mathrm{nm}$, so that incident laser radiation with comparable field strengths could produce a bright source of microwave or $\mathrm{THz}$ radiation.

\subsection{Carbon Nanotips-BaSed Field EmitTers}

Due to the technological importance of carbon fibers, there has been a growing interest towards understanding the mechanism of the field electron emission from these fibers under the influence of an applied electrical field. Using carbon fibers as cathodes brings several benefits such as offering the ability to work in a relatively high pressure environment (about $10^{-6} \mathrm{mbar}$ ), simplicity of manufacturing the emitter, high current stability and long emitter durability. Such cathodes are made either from carbon fibers or from other carbon-based materials. Carbon fiber emitters can be produced by electrolytic etching technique, where a 0.1 Molarit of sodium hydroxide solution ( $250 \mathrm{ml}$ of distal water with $2 \mathrm{~g}$ of $\mathrm{NaOH}$ ) is used [8]. This etching process could be controlled by choosing a suitable etching current. The etching process is started after dipping the tip in the solution by about $2 \mathrm{~mm}$ and using a power supply to increase the voltage until a certain initial current of about $30 \mu \mathrm{A}$ is reached. The chosen etching current produces sharp tips at the liquid 
surface which are afterwards being ultrasonically cleaned using an ultrasonic cleaning device and mounted in a standard field emission microscopy (FEM) with a tip screen distance of $10 \mathrm{~mm}$. The anode is formed as phosphored screen to allow recording of the emission images.

The FEM was evacuated to ultra high vacuum (UHV) conditions using rotary pump that produce pressures of about $10^{-3}$ mbar and a diffusion pump system in addition to a liquid nitrogen (LN2) trap that lead to finally reaching a base pressure of about $10^{-9} \mathrm{mbar}$. Then, the tips received sample conditioning which consists of an initial baking of the system for 12 hours at $170{ }^{\circ} \mathrm{C}$, a follow up baking of the system for 12 hours, thermal relaxation for 12 hours at $170{ }^{\circ} \mathrm{C}$, and finally cooling the sample by liquid nitrogen - while studying emission behavior [8]. This allowed the recording of the effects of these conditioning processes on carbon fiber tips.

To record the emission behavior, the voltage applied from extra high tension (EHT) to the tip is slowly increased until the emission current rises to about one microampere on the picoammeter device, and then the voltage is slowly decreased until the emission current was vanished. Within this range, a linear Fowler-Nordheim plot is expected. Figure 16 presents the scanning electron micrograph of a produced very sharp carbon fiber tip at about 10,000 x magnification.

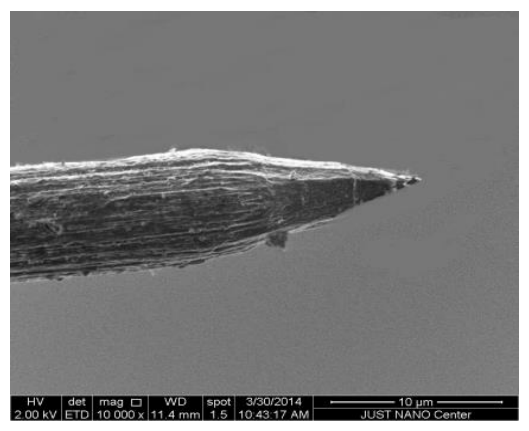

Figure 16. Scanning electron micrograph of a very sharp carbon fiber tip at $10,000 \times$ magnification.

The apex radii of carbon fiber tips have been measured as the average of the graphically bestfitting circles. The latter ones are used to interpret the experimental data and extract relevant information such as the apex radii. During the experiments, electronic emission images have been recorded by a standard digital camera to study the spatial distribution and stability of the emission current. Stability as well as brightness are important factors for judging the quality of the electron source for practical applications. During the sample conditioning treatment, it was discovered that there were statistical variations in the electronic emission of the various tip microemitters under the corresponding UHV conditions. One of the very sharp carbon fiber tips has been tested during sample conditioning treatment [8] where the apex radius of this tip was around $57 \mathrm{~nm}$.

Figures 17-20 show the emission characteristics that are derived as the I-V characteristics and the corresponding Fowler-Nordheim plots.
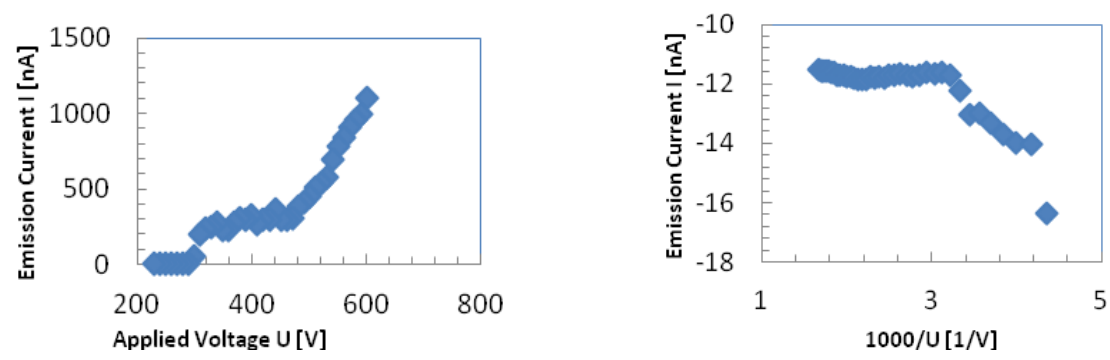

Figure 17. The I-V characteristics (left) and FN plot (right) of very sharp tip after initial baking for 12 hours at temperature of $170^{\circ} \mathrm{C}$. 

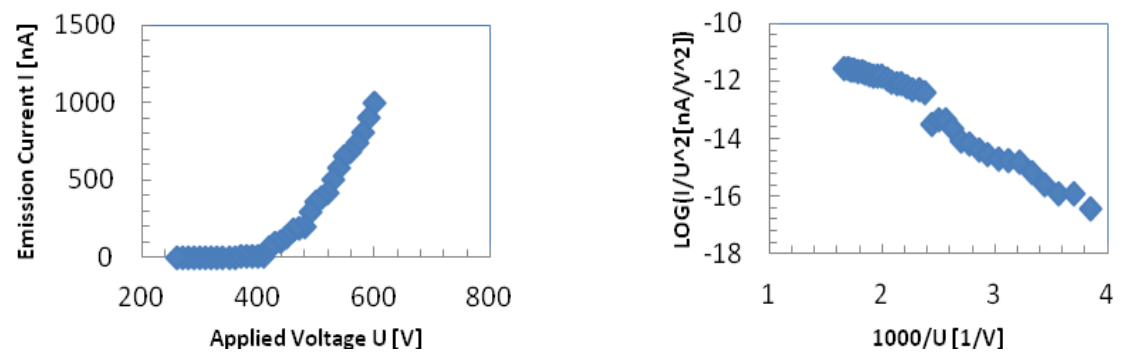

Figure 18. The I-V characteristics (left) and FN plot (right) of very sharp tip after follow-up baking for 12 hours at temperature of $170^{\circ} \mathrm{C}$.
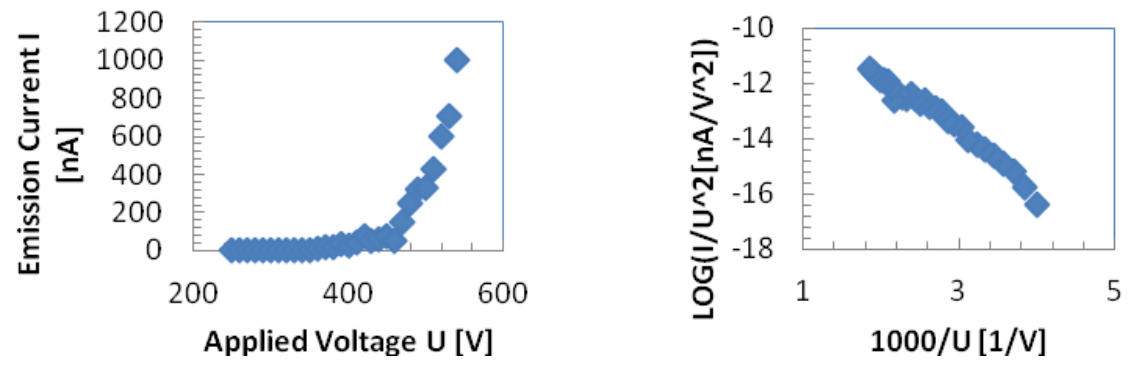

Fig. 19. The I-V characteristics (left) and FN plot (right) of very sharp tip after thermal relaxation process for 12 hours.
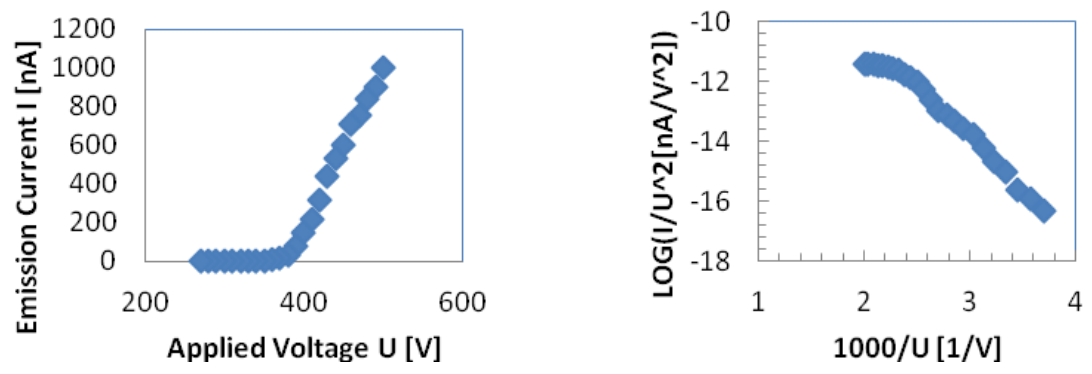

Fig. 20. The I-V characteristics (left) and FN plot (right) of very sharp tip during cooling process.

After initial baking, as was shown in Fig. 17, the FN plot shows a non-linear behavior and emission current was unstable. The follow up baking, as shown in Fig. 18, shows a disconnected plot because the voltage drops from $66 \mathrm{~V}$ to $5.5 \mathrm{~V}$ where this drop is due to hysterical current. After the sample conditioning, the emission current stability becomes much higher as shown in Figs. 19 and 20.

As previously mentioned, the presented background and fundamentals in this section with regards to carbon nanotubes and nanotips and their corresponding field-emission will be further utilized, in addition to the basic background on lattice networks that was presented in Sections 2 and 3, within the follow-up works in the second and third parts of the article for designing concurrent nano-based three-dimensional lattice networks, where the new implementation of ternary Galois functions using carbon field-emission switching devices will be performed. 


\section{CONCLUSIONS}

Fundamentals of concurrent processing using three-dimensional lattice networks are presented, and basic characteristics of carbon-based field-emission are also shown. This includes the newly investigated Fowler-Nordheim and I-V characteristics for carbon nanotip-based field emitters. The presented basics in this first part will be utilized within the second and third parts of the article for the synthesis of nano-based three-dimensional networks to implement ternary Galois functions in three-dimensions using carbon field-emission switching devices that are based on the presented carbon nanotube and nanotip field-emission characteristics.

The two-to-one controlled switch is a basic building block in switch logic, which is proven to be of fundamental practical importance in cost-effective modern logic design within wide variety of circuits and systems. The concept of switch logic is that logic circuits are implemented as combination of switches rather than a combination of gates as in gate logic, where it has been shown that this type of synthesis possesses direct one-to-one mapping from the design layer into physical layer for wide range of applications including lattice networks. As previously stated, the presented field-emission basics in this first part will be further utilized in the second and third parts of the article which form new and interesting design directions via the synthesis of emission-based controlled-switching within concurrent three-dimensional lattice systems.

\section{ACKNOWLEDGEMENT}

This research was performed during sabbatical leave in 2019-2020 granted to the author from The University of Jordan and spent at Isra University, Jordan.

\section{REFERENCES}

[1] S. B. Akers, "A rectangular logic array," IEEE Transactions on Computers, Vol. C-21, pp. 848-857,1972.

[2] A. N. Al-Rabadi, Reversible Logic Synthesis: From Fundamentals to Quantum Computing, Springer-Verlag, New York, 2004.

[3] A. N. Al-Rabadi, "Three-dimensional lattice logic circuits, Part I: fundamentals," Facta Universitatis - Electronics and Energetics, Vol. 18, No. 1, pp. 1 - 13, 2005.

[4] A. N. Al-Rabadi, "Carbon nano tube (cnt) multiplexers for multiple-valued computing," Facta Universitatis - Electronics and Energetics, Vol. 20, No. 2, pp. 175-186, 2007.

[5] A. N. Al-Rabadi, "New dimensions in non-classical neural computing, Part II: quantum, nano, and optical," International Journal of Intelligent Computing and Cybernetics, Emerald, Vol. 2, No. 3, pp. 513-573, 2009.

[6] A. N. Al-Rabadi, Carbon NanoTube (CNT) Multiplexers, Circuits, and Actuators, United States Patent and Trademark Office, Patent No. US 7,508,039 B2, U.S.A., 2009.

[7] A. N. Al-Rabadi, "Circuits for $m$-valued classical, reversible and quantum optical computing with application to regular logic design," Int. Journal of Intelligent Computing and Cybernetics (IJICC), Emerald, Vol. 2, No. 1, pp. 52 - 101, 2009.

[8] S. Alnawasreh, M. S. Mousa, and A. N. Al-Rabadi, "Investigating the effects of sample conditioning on nano-apex carbon fiber tips for efficient field electron emission," Jordan J. Physics, Vol. 8, No. 1, pp. 51-57, 2015.

[9] K. Alonso and M. J. Hagmann, "Comparison of three different methods for coupling of microwave and terahertz signals generated by resonant laser-assisted field emission," Journal of Vacuum Science \& Technology B: Microelectronics and Nanometer Structures, Vol. 19, No. 1, pp. 68-71, 2001.

[10] G. Amaratunga, "Watching the nanotube," IEEE Spectrum, pp. 28-32, 2003.

[11] J. M. Bonard, K. A. Dean, B. F. Coll, and C. Klinke, "Field emission of individual carbon nanotubes in the scanning electron microscope," Physical Review Letters, 89(19):197602-1:4, 2002.

[12] M. Brugat, M. S. Mousa, E. P. Sheshin, and M. J. Hagmann, "Measurement of field emission current variations caused by an amplitude modulated laser," Materials Science and Engineering A, 327(1):7-15, 2002.

[13] R. E. Bryant, "Graph-based algorithms for Boolean functions manipulation," IEEE Transactions on Computers, Vol. C-35, No. 8, pp. 667-691, 1986.

[14] P. J. Burke, "Luttinger liquid theory as a model of the gigahertz electrical properties of carbon nanotubes," IEEE Transactions on Nanotechnology, 1(3):129-144, 2002.

[15] H. F. Cheng, Y. S. Hsieh, Y. C. Chen, and I. N. Lin, "Laser irradiation effect on electron field emission properties of carbon nanotubes," Diamond and Related Materials, 13(4-8):1004-1007, 2004.

[16] P. G. Collins and P. Avouris, "Nanotubes for electronics," Scientific American, pp. 62-69, 2000.

[17] P. G. Collins, M. S. Arnold, and P. Avouris, "Engineering carbon nanotubes and nanotube circuits using electrical breakdown," Science, Vol. 292, 2001.

[18] V. Derycke, R. Martel, J. Appenzeller, and P. Avouris, "Carbon nanotube inter- and intramolecular logic gates," Nano Letters, Vol. 0, No. 0, A - D, 2001.

[19] E. Drexler, Engines of Creation: the Coming Era of Nanotechnology, Anchor Books, 1986. 
[20] R. G. Forbes, "Extraction of emission parameters for large-area field emitters, using a technically complete FowlerNordheim-type equation," Nanotechnology, IOP Publishing, 23(9), 2012.

[21] R. H. Fowler and L. W. Nordheim, "Electron emission in intense electric fields," Proceedings of the Royal Society A: Mathematical, Physical and Engineering Sciences, 119(781): 137-181, 1928.

[22] T. Fujieda, K. Hidaka, M. Hayashibara, T. Kamino, Y. Ose, H. Abe, T. Shimizu, and H. Tokumoto, "Direct observation of field emission sites in a single multiwalled carbon nanotube by Lorenz microscopy," Japanese Journal of Applied Physics, 44(4A):1661-1664, 2005.

[23] D.H. Green, "Families of Reed-Muller canonical forms," International Journal of Electronics, No. 2, pp. 259-280, 1991.

[24] M. J. Hagmann and M. S. Mousa, "Time-dependent response of field emission by single carbon nanotubes," Jordan Journal of Physics, 1(1): 1-7, 2008.

[25] P. Hommelhoff, Y. Sortais, A. Aghajani-Talesh, and M. A. Kasevich, "Field emission tip as a nanometer source of free electron femtosecond pulses," Physical Review Letters, 96(7):077401-1:4, 2006.

[26] J. Jiao, E. Einarsson, D. W. Tuggle, L. Love, J. Prado, and G. M. Coia, "High-yield synthesis of carbon coils on tungsten substrates and their behavior in the presence of an electric field," J. Materials Research, Vol. 18, No. 11, pp. 2580-2587, 2003.

[27] M. G. Karpovsky, Finite Orthogonal Series in the Design of Digital Devices, Wiley, New York, 1976.

[28] Z. Kohavi, Switching and Finite Automata Theory, McGraw-Hill, New York, 1978.

[29] K. Likharev, "Electronics below $10 \mathrm{~nm}$," in: Nano and Giga Challenges in Microelectronics, pp. 27-68, Elsevier, 2003.

[30] A. N. Obraztsov, I. Pavlovsky, A. P. Volkov, E. D. Obraztsova, A. L. Chuvilin, and V. L. Kuznetsov, "Aligned carbon nanotube films for cold cathode applications," Journal of Vacuum Science \& Technology B, 18(2): 1059-1063, 2000.

[31] K. C. Smith, "Prospects for VLSI technologies in MVL," Booklet of Ultra Large Scale Integration (ULSI) Workshop, p.4, Boston, Massachusetts, 2002.

[32] R. S. Stankovic, J. Astola, and C. Moraga, "Fast Fourier transforms on finite groups as a method in synthesis for regularity," Journal of Multiple-Valued Logic and Soft Computing, 23(5-6), pp. 463-483, 2014.

[33] J. A. Stratton, Electromagnetic Theory, McGraw-Hill, New York, 1968.

[34] D. Tomanek, R. Enbody, K. Young-Kyun, and M. W. Brehob, "Nanocapsules containing charged particles, their uses and methods of forming the same," U.S. Patent, Publication No.: US 2002/0027819 A1, 2002.

[35] S. N. Yanushkevich, V. P. Shmerko, and S. E. Lyshevski, Logic Design of NanoICs, CRC Press, 2004.

[36] T. Zhang, J. Chang, W. Su, X.-L. Zhou, and X. Jia, "Thermoelectric properties of antimony films: roles of oxidation and topological quantum state," Nanotechnology, 31(48), 2020.

[37] L. Zhang, S. Fahad, H.-R. Wu, T.-T. Dong, Z.-Z. Chen, Z.-Q. Zhang, R.-T. Liu, X.-P. Zhai, X.-Y. Li, X. Fei, Q.-W. Song, Z.-J. Wang, L.-C. Chen, C.-L. Sun, Y. Peng, Q. Wang, and H.-L. Zhang, "Tunable nonlinear optical responses and carrier dynamics of two-dimensional antimonene nanosheets," Nanoscale Horizons, 5(10), pp. 1420-1429, 2020.

[38] Z. Zhu, J. Guan, and D. Tomanek, "Strain-induced metal-semiconductor transition in monolayers and bilayers of gray arsenic: A computational study," Physical Review B, 91, 161404(R), 2015.

\section{AUTHOR}

Anas N. Al-Rabadi is currently a Professor in the Department of Computer Engineering at The University of Jordan. He received his Ph.D. in Computer Design and Advanced Logic Synthesis from the Electrical and Computer Engineering Department at Portland State University in 2002, received his M.Sc. in Feedback Control Systems and Power Electronics Systems Design from the Electrical and Computer Engineering Department at Portland State University in 1998, and was a Research Faculty at the Office of Graduate Studies and Research (OGSR) at Portland State University. He is the author of the first comprehensive graduate-level book and the first published title on Reversible Logic Synthesis, Reversible Logic Synthesis: From Fundamentals to Quantum Computing (Springer-Verlag, 2004). Currently, Prof. AlRabadi is the author of more than 130 international scholarly articles that are published in international books, book chapters, journals and conferences, in addition to a registered U.S.A. nanotechnology patent. His current research includes parallel and distributed computing, systolic architectures, regular circuits and systems, reversible logic, quantum computing, multiple-valued logic, soft computing and computational intelligence, machine learning, optical computing, reconstructability analysis, signal processing, testing and design for testability, nanotechnology, robotics, optimal robust control, and digital error-control coding. 